\title{
Force-based higher-order beam element with flexural-shear-torsional interaction in 3D frames. Part II: Applications
}

\author{
João P. Almeida ${ }^{\mathrm{a}, \mathrm{b}, *}$, António A. Correia ${ }^{\mathrm{a}, \mathrm{c}, 1}$, Rui Pinho ${ }^{\mathrm{d}, 2}$ \\ ${ }^{\text {a }}$ EUCENTRE, Pavia, Italy \\ ${ }^{\mathrm{b} C}$ Current affiliation: École Polytechnique Fédérale de Lausanne, Switzerland \\ ' Current affiliation: Department of Structures, National Laboratory for Civil Engineering (LNEC), Lisbon, Portugal \\ ${ }^{\mathrm{d}}$ Department of Civil Engineering and Architecture, University of Pavia, Italy
}

\section{A R T I C L E I N F O}

\section{Article history:}

Received 25 February 2014

Revised 31 August 2014

Accepted 6 October 2014

Available online 17 December 2014

\section{Keywords:}

Beam element

Force-based

Higher-order

Flexural-shear-torsional interaction

Warping

Timoshenko

\begin{abstract}
A B S T R A C T
The specific features of the proposed force-based formulation derived in the companion paper, which is applied for the first time to higher-order beam theories, are herein thoroughly validated. Introductory numerical examples illustrate the influence of mesh refinement, boundary conditions, and slenderness ratios for isotropic linear elastic response. Specific higher-order effects-unique to the developed element-are then suitably interpreted, as well as the formulation appropriateness to consider distributed loads and to model three-dimensional behaviour, which is verified with solid finite element analyses. Extensive comparisons against existing proposals, namely other refined higher-order beam theories, emphasize the performance of the proposed approach. Finally, the nonlinear response of the element with a multiaxial $\mathrm{J} 2$ linear plasticity material model is analysed, highlighting its advantages in relation to a classical force-based Euler-Bernoulli beam using a one-dimensional plastic material model with kinematic hardening.
\end{abstract}

(c) 2014 Elsevier Ltd. All rights reserved.

\section{Introduction}

The transfer of the advantages of force-based formulations to higher-order beam theories (HOBTs), which to the authors' knowledge was proposed for the first time in the companion paper [1], is herein investigated from the application viewpoint. The theoretical background previously introduced indicated that the novel element satisfies an advanced form of equilibrium, in-between that of the classical Timoshenko beam theory and the complete threedimensional equilibrium, which is herein confirmed with numerical examples.

Although the last example of this manuscript showcases an application of the present formulation to a structural member behaving nonlinearly, there are specificities accruing from the fact that a HOBT is considered in association with a flexibility formulation that justify an exhaustive inspection of the model performance with isotropic linear elastic material behaviour. Typically, the advantages of force-based approaches, mentioned in the com-

\footnotetext{
* Corresponding author at: EPFL ENAC IIC EESD, GC B2 484 (Bâtiment GC), Station 18, 1015 Lausanne, Switzerland.

E-mail addresses: joao.almeida@epfl.ch (J.P. Almeida), aacorreia@lnec.pt (A.A. Correia), rui.pinho@unipv.it (R. Pinho).

1 Address: LNEC-DE/NESDE, Av. do Brasil 101, 1700-066 Lisbon, Portugal.

2 Address: c/o EUCENTRE, Via Ferrata 1, 27100 Pavia, Italy.
}

panion paper, imply that very coarse meshes of beam elements based on Euler-Bernoulli theory can be used to model structural members. Frequently, one finite element per member can be used even in the presence of material nonlinear behaviour and span loads. Such features are not necessarily applicable in a straightforward way in the present setting, in view of the special higher-order effects that arise due to specification of distinct sets of boundary conditions (BCs), which differ from any previous results obtained with displacement-based formulations; therefore, they should be thoroughly assessed, as well as the influence of mesh refinement, usually known as h-refinement. An insightful interpretation of these and other salient features of the model, as well as a comparison against classical and state-of-the-art proposals, restrict the numerical applications herein presented to the case of solid rectangular cross-sections. However, as pointed out in the companion paper, the proposed theory can be extended to members with more general cross-sectional geometries, which will be addressed in forthcoming publications.

The first group of numerical examples in Section 2 analyse the response of the element under nodal in-plane loading, comparing its response against the results of Euler-Bernoulli beam theory (EBBT) and other HOBTs, namely the one from Heyliger and Reddy [2]. This serves as a motivation to analyse in detail the specific higher-order effects of the proposed flexibility approach, which is carried out in Section 3. The subsequent examples of Sections 4 
and 5 evaluate respectively the suitability of the formulation to simulate the effects of span loads and three-dimensional behaviour, which is validated with solid finite element models. A comparison with other refined HOBTs, particularly the one from Carrera et al. [3,4], follows in Section 6. Finally, the performance of the element under nonlinear material behaviour, which will hopefully open the door to many future applications, is examined in Section 7.

The numerical examples were run in the structural analysis code SAGRES (Software for Analysis of GRadient Effects on Structures), which was developed by the authors using the MATLAB platform [5]. Further details on its numerical implementation can be found in Section 7.1. Base or derived units of the International System of Units (SI) are used.

From the 38 cross-sectional displacement modes considered in the formulation, a chosen illustrative set of 15 modes are represented in Fig. 1. Modes 1 through 8 are the first-order ones, being composed of the six modes corresponding to classical Timoshenko beam theory, followed by two related to warping and distortion. Out of the remaining 30 higher-order displacement modes, defined in the companion paper based on normalised Legendre polynomials [1], seven are also depicted: modes 9 and 11 are the first describing a nonlinear displacement variation throughout the cross-section, parabolic along the in-plane axes; modes 14 and 15 are exemplificative of the way in which significant physical phenomena associated with cross-sectional in-plane deformation (e.g., Poisson's effect or confinement in reinforced concrete beams) may be accounted for in the present formulation; modes 21 and 31 complement modes 1,5 and 11 as cross-sectional representations of the initial five Legendre polynomials; finally, mode 36 is a sample of those modes featuring a product of polynomials of different degree along the two orthogonal cross-sectional coordinate axes.

The description above should render clear that the relatively large number of degrees of freedom (dof) of the present formulation allows to explicitly consider an extended range of displacement modes that other proposals, directed to different applications, prefer to neglect or condense out. For example, recent theories that have been developed within the relatively similar framework of the Hellinger-Reissner variational principle (Wackerfuss and Gruttmann [6]) opt to focus on the out-of-plane warping effects. The latter can be described by one extra parameter (Kumar et al. [7]), which adds to the six dof at each node, or by internal degrees of freedom approximating the cross-sectional warping that are eliminated at the element level by static condensation (Wackerfuss and Gruttmann [8]).

\section{Cantilever with in-plane tip load}

In the past, other researchers (e.g., Eisenberger [9]) have used as a benchmark test the case of a rectangular cantilevered beam subjected to an end load $p=100[\mathrm{~N}]$, and the following properties: width $b=1[\mathrm{~m}]$, Young's modulus $E=29,000\left[\mathrm{~N} / \mathrm{m}^{2}\right]$, Poisson's ratio $v=0.3$. This problem, originally analysed by Heyliger and Reddy [2] and herein considered again, was solved for different values of length $L=\{12,40,80,160\} \quad[\mathrm{m}]$ and cross-sectional height $h=\{1,12\}[\mathrm{m}]$.

The finite element introduced by Heyliger and Reddy [2] is based on the planar third-order beam theory proposed by Levinson [10], which has the following displacement field:

$\mathbf{u}(x, z)=\left[\begin{array}{l}u_{x} \\ u_{z}\end{array}\right]=\left[\begin{array}{c}u_{x 0}+z \theta_{y}+z^{3} \phi \\ u_{z 0}\end{array}\right]=\left[\begin{array}{cccc}1 & 0 & z & z^{3} \\ 0 & 1 & 0 & 0\end{array}\right]\left[\begin{array}{c}u_{x 0} \\ u_{z 0} \\ \theta_{y} \\ \phi\end{array}\right]$

In order to guarantee vanishing shear strains on the top and bottom free surfaces of the beam, a value of the cross-sectional warping intensity $\phi=-4 / 3 h^{2}\left(\theta_{y}+u_{z 0}^{\prime}\right)$ is derived, yielding the following modified expression:

$\mathbf{u}(x, z)=\left[\begin{array}{ccc}1 & z^{3}\left(-\frac{4}{3 h^{2}}\right) \frac{\partial}{\partial x} & z+z^{3}\left(-\frac{4}{3 h^{2}}\right) \\ 0 & 1 & 0\end{array}\right]\left[\begin{array}{c}u_{x 0} \\ u_{z 0} \\ \theta_{y}\end{array}\right]=\mathbf{U}(z) \mathbf{d}(x)$

As discussed in the introduction of the companion paper, the previous displacement field has been used in a number of works up to the present day, either related to beam theory (Reddy [11])
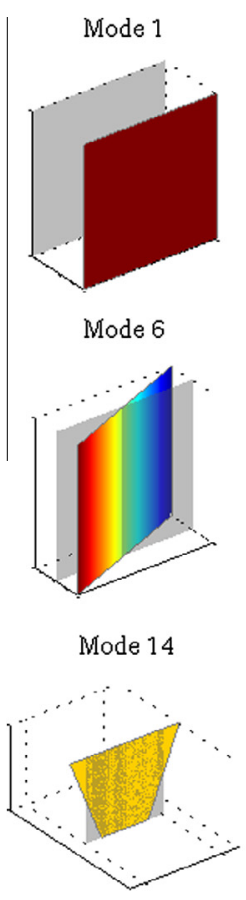

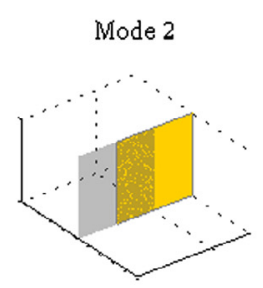

Mode 7
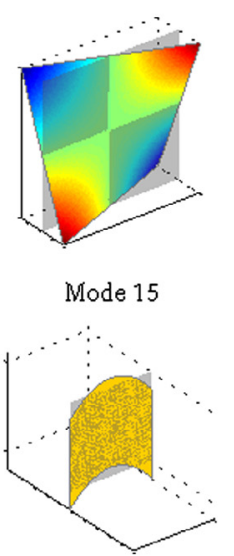

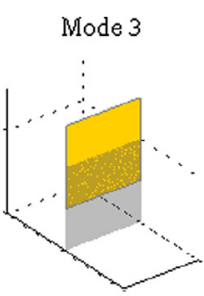

Mode 8
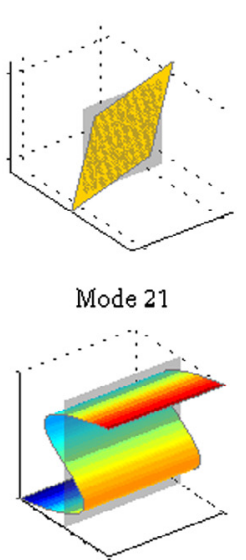

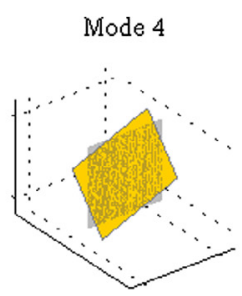

Mode 9
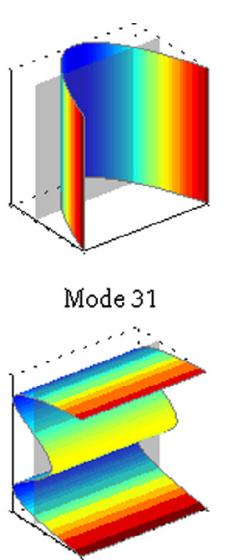

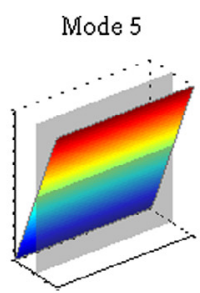

Mode 11
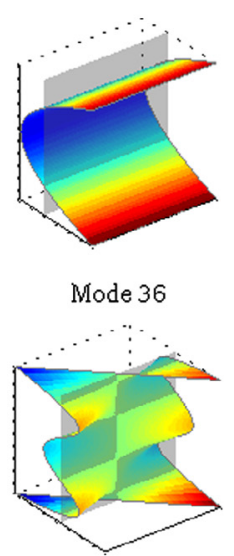

Fig. 1. Illustrative orthogonal displacement modes for a square cross-section. 
or plate theory (Reddy $[12,13])$. The formulation by Heyliger and Reddy [2] is a displacement-based approach-using Lagrange and Hermite interpolation functions-which stands out as perhaps the most fundamental difference in relation to the proposed theory. Other advantageous features of the latter, such as the definition of the generalised forces and relevant orthogonality properties, can be found in the companion paper [1]. The following comparisons with the present beam theory are obviously restricted to the scope of applicability of the above equation, which is planar loading; the full three-dimensional capabilities of the proposed force-based HOBT will be illustrated in Section 5 of this document.

\subsection{Mesh refinement and bounding of the solution}

Members wherein flexural deformation is expected to be predominant, that is, with cross-sectional height $h=1$ [m], are studied first. Fig. 2 depicts the comparison between the tip displacements given by the proposed HOBT and that of Heyliger and Reddy [2]. The results are normalised with respect to those provided by the EBBT, which for the assumed geometric and loading conditions (prismatic element, linear elastic behaviour and nodal loads), always provide the same solution; this holds independently of the number of elements $(N)$ and type of formulation (displacement-based or force-based) [14].

A number of remarks should now be made about these introductory results. To start with, the apparent underperformance of HOBTs with respect to EBBT, even if slight, deserves further explanations. As a matter of fact, the asymptotic response in the figure below shows that both HOBTs are affected by specific effects-which will later be analysed in detail-that vanish with mesh refinement, indicating that they correspond to undesired features of higher-order formulations rather than to physical phenomena, such as shear deformation. Actually, the latter is barely discernible for the slenderness ratios $L / h$ herein considered,

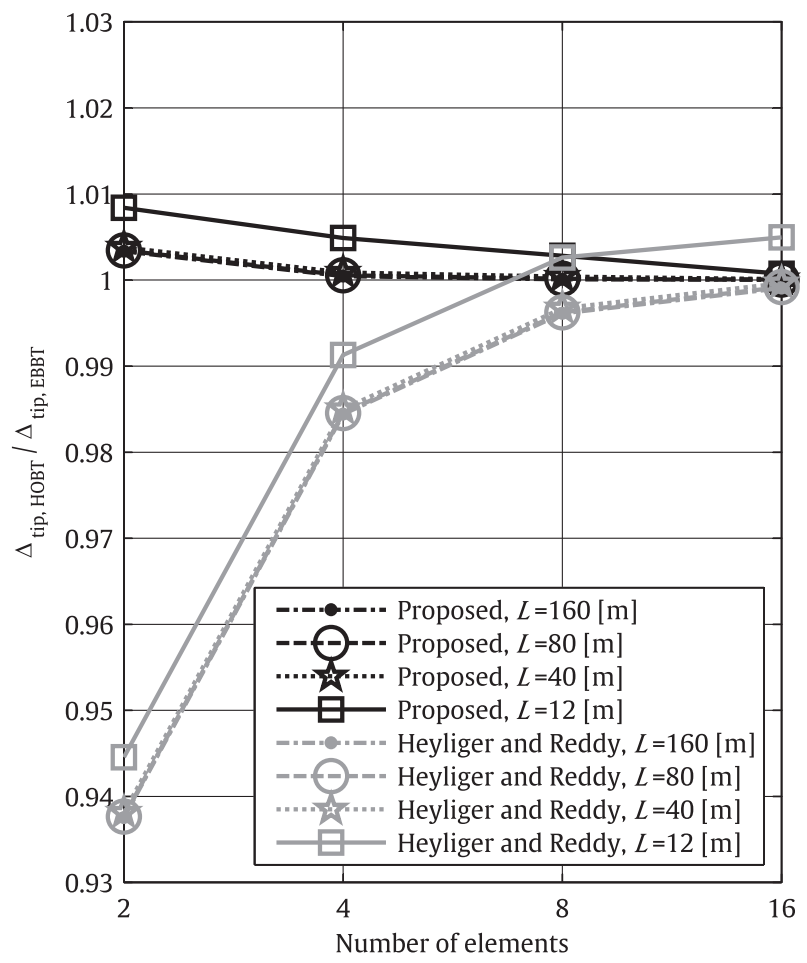

Fig. 2. Comparison of the proposed theory and that of Heyliger and Reddy [2], relative to the results provided by the Euler-Bernoulli theory, for a cross-sectional height of $h=1[\mathrm{~m}]$ and concentrated tip load. as evidenced by the close-to-unity results of the several 16 -element meshes. The only exception is the performance of the case with $L / h=12$ in the formulation of Heyliger and Reddy [2], which is however limited to a deviation of less than $0.5 \%$ in relation to the EBBT.

Another important observation is related to the bounding of the solution. As mentioned earlier, the most common type of beam-column modelling approach is the so-called stiffness or displacement-based formulation (sometimes also known as pure compatibility model in the literature), which makes use of compatible displacement interpolation functions and the principle of virtual work, also known as principle of virtual displacements. On the other hand, the flexibility or force-based formulations (which also go by the name of pure equilibrium models) are built on the derivation of self-equilibrated stress interpolation functions and the principle of complementary virtual work or principle of virtual forces.

Equivalently, the former class of models can be obtained through the application of the theorem of potential energy, while the latter can be retrieved with the theorem of complementary energy (Washizu [15]). They can also be derived by other equivalent variational approaches or by the more general methodology presented by Teixeira de Freitas et al. [16], which builds on the first principles of mechanics to obtain the corresponding duality principles. The seminal work by de Veubeke [17] was the first to demonstrate that the standard potential energy formulation provides a lower-bound to the energy of the system, while the complementary energy formulation provides an upper-bound. As far as the authors are aware of, Fig. 2 is the first in the literature to provide evidence of the previous principle regarding HOBTs. Nevertheless, this bounding property can only be fully exploited if both HOBTs use the same higher-order modes of deformation, which is not the case of Fig. 2. In that sense, Section 6 will provide fairer comparisons.

The previous note relates to the explanation for the apparently distinct asymptotic values of each HOBT, as the mesh refines, in the case of $L / h=12 / 1$. Such behaviour is even clearer in Fig. 3, which depicts the results for a cross-section of height $h=12$ [m]. The underlying reason is primarily the fact that the level of equilibrium verified by the proposed theory is much more advanced than the degree of compatibility respected by the theory of Heyliger and Reddy [2]. This is directly attributable to the larger number and enriched shapes of the cross-sectional displacement modes considered in the proposed theory, which yields governing differential equations describing more evolved structural element behaviour.

It should be noted that the strain energy of a finite element developed through a hybrid method may be an upper- or lowerbound, however it is always bounded by that of a compatible model using the same type of inter-element boundary displacements and that of an equilibrium model using the same type of interior stresses [18].

\subsection{Accuracy}

It is visible, namely for 2, 4 and 8-element meshes, that the results obtained with the proposed HOBT are more accurate than those of Heyliger and Reddy [2] with an identical number of elements. One could be tempted to attribute such differences, once again, to the larger number of higher-order modes considered in the proposed theory; however, as mentioned in the introduction, such improvement can in fact be mainly ascribed to the specific advantages of the devised formulation (force-based versus displacement-based) when coarse meshes are employed.

Fig. 4 provides further evidence of the previous observation, as well as a depiction of the proposed model accuracy. The plot shows the relative error of the tip displacement given by both theories, 


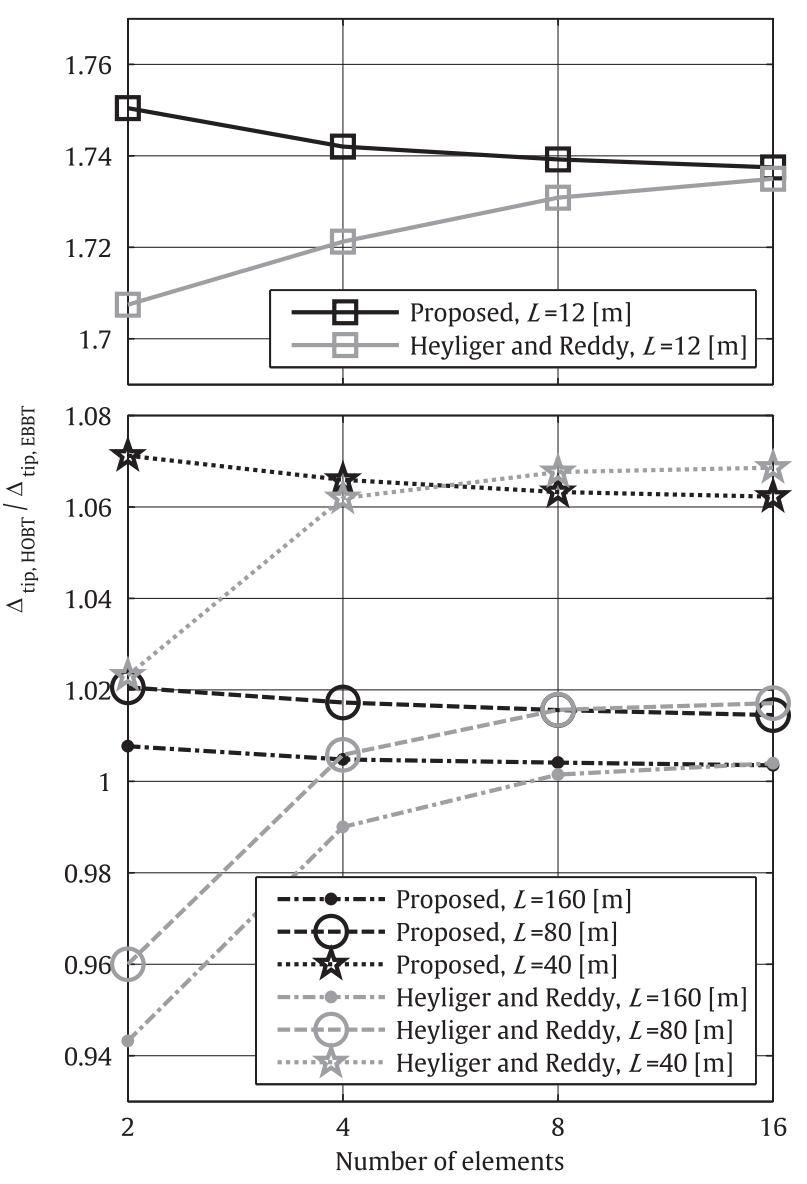

Fig. 3. Comparison of the proposed theory and that of Heyliger and Reddy [2], relative to the results provided by the Euler-Bernoulli theory, for a cross-sectional height of $h=12[\mathrm{~m}]$ and concentrated tip load.

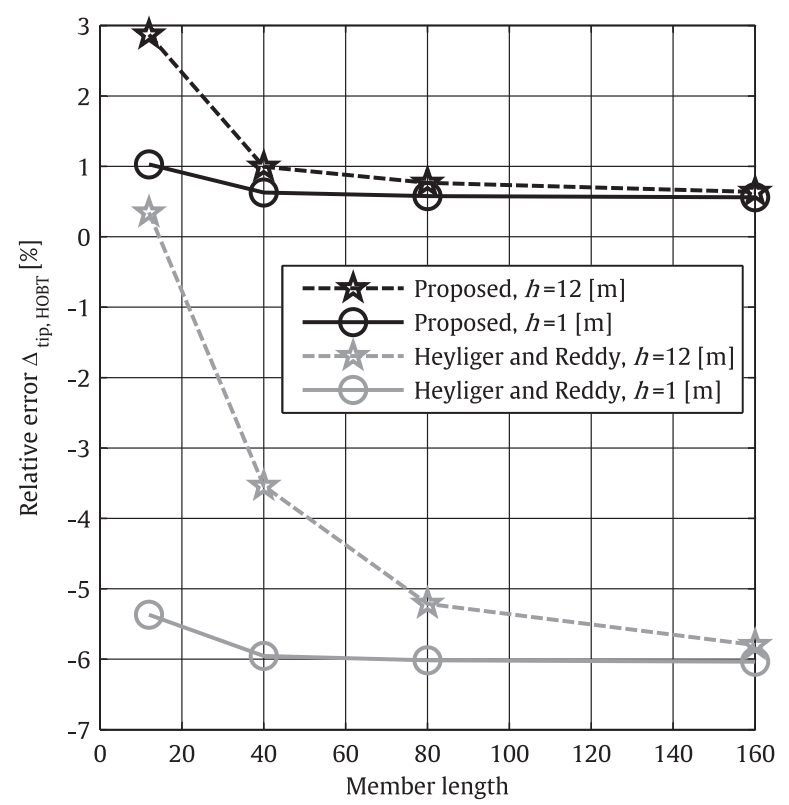

Fig. 4. Relative error of the tip displacement, for the case of two-element meshes $(N=2)$, assuming as exact the response given by a solid finite element mesh.

using a coarse mesh of two elements $(N=2)$. The 'exact' response was estimated via 3D models with refined meshes of solid finite elements $[19,20]$. At the clamped extremity the nodes were fully restrained, whilst on the opposite end transversal forces corresponding to a constant shear stress distribution were imposed in all boundary nodes. The excellent element performance illustrated in Fig. 4 is backed up again in Section 6, which deals with the comparison with other refined HOBTs employing a displacement-based approach.

Fig. 3 also shows that, when $L / h=160 / 12$, the displacement response ratio is roughly and unsurprisingly similar to the case of Fig. 2 with an approximately similar slenderness ratio of $L / h=12 / 1$. As the ratio between the beam length and the cross-sectional height progressively decreases, the relevance of shear deformation and other physically meaningful three-dimensional effects increase. That is evident from the results with the several 16-element meshes, which provide tip displacements significantly higher than those of the classical elementary beam theory. In particular, such deviation increases pronouncedly from approximately $6.5 \%$ to $73.5 \%$ as the slenderness ratio reduces from $L / h=40 / 12=3.33$ to $L / h=12 / 12=1$. The following section shows in more detail the physical phenomena reproduced by the present model, relating them with the boundary conditions and higher-order effects specific to the proposed formulation.

\section{Higher-order effects and boundary conditions}

As observed by other researchers (Bickford [21]; Prathap et al. [22]), HOBTs are subjected to specific effects that require careful interpretation and a study of their influence on the accuracy of the results. They are intrinsically related to the boundary conditions (BCs) assumed, which for this model consist of a combination of imposed generalised nodal displacements and/or forces, in a total of 76 (38 at each extremity) as derived by Correia et al. [1]. The special nature of such effects-when applied together with a flexibility formulation-naturally asks for a careful analysis in order to expose this yet undisclosed behaviour.

\section{1. 'Totally fixed - free'}

The results shown in the previous paragraph were obtained, for the proposed theory, with the following set of BCs: on the clamped extremity, all the 38 generalised displacements were restrained, and on the free end-where the tip force is applied-the 38 generalised forces were controlled. Out of the latter, 37 were kept equal to zero, while on the degree of freedom corresponding to the shear force a value of 100 was imposed. For future reference, this set of BCs is denoted by the acronym TFF, which stands for 'totally fixed - free'. The question that arises is how the set of conditions TFF relate to the actual physical behaviour of the member, and if there are more suitable BCs to describe it. A critical inspection of the performance of the proposed beam theory is required to answer such query, which differs from all other HOBTs proposed to date. The latter goal can be effectively achieved by analysing the response of the slender beam with $L=160[\mathrm{~m}]$ and $h=1[\mathrm{~m}]$, wherein flexural deformations are expected to be absolutely predominant.

For the current loading case the behaviour of the element is governed by the differential equilibrium equations shown in system (3) of Appendix A of Correia et al. [1]. Hence the generalised stress-resultants therein included are expected to play a role in the response. The evolution of $V_{z}$ and $M_{y}$ match exactly the diagrams that one would obtain from the application of classical Euler-Bernoulli or Timoshenko beam theories, i.e., respectively constant (equal to $100[\mathrm{~N}]$ ) and linear (from 0 at the tip to $-16,000[\mathrm{~N} \cdot \mathrm{m}]$ at the fixed end). This feature is appealing since it corresponds to the results expected from common engineering reasoning. Additionally, the values of $N^{*, 03}$ and $V_{z}^{*, 03}$ are also relevant, their evolution being traced in Fig. 5 for h-refinements 

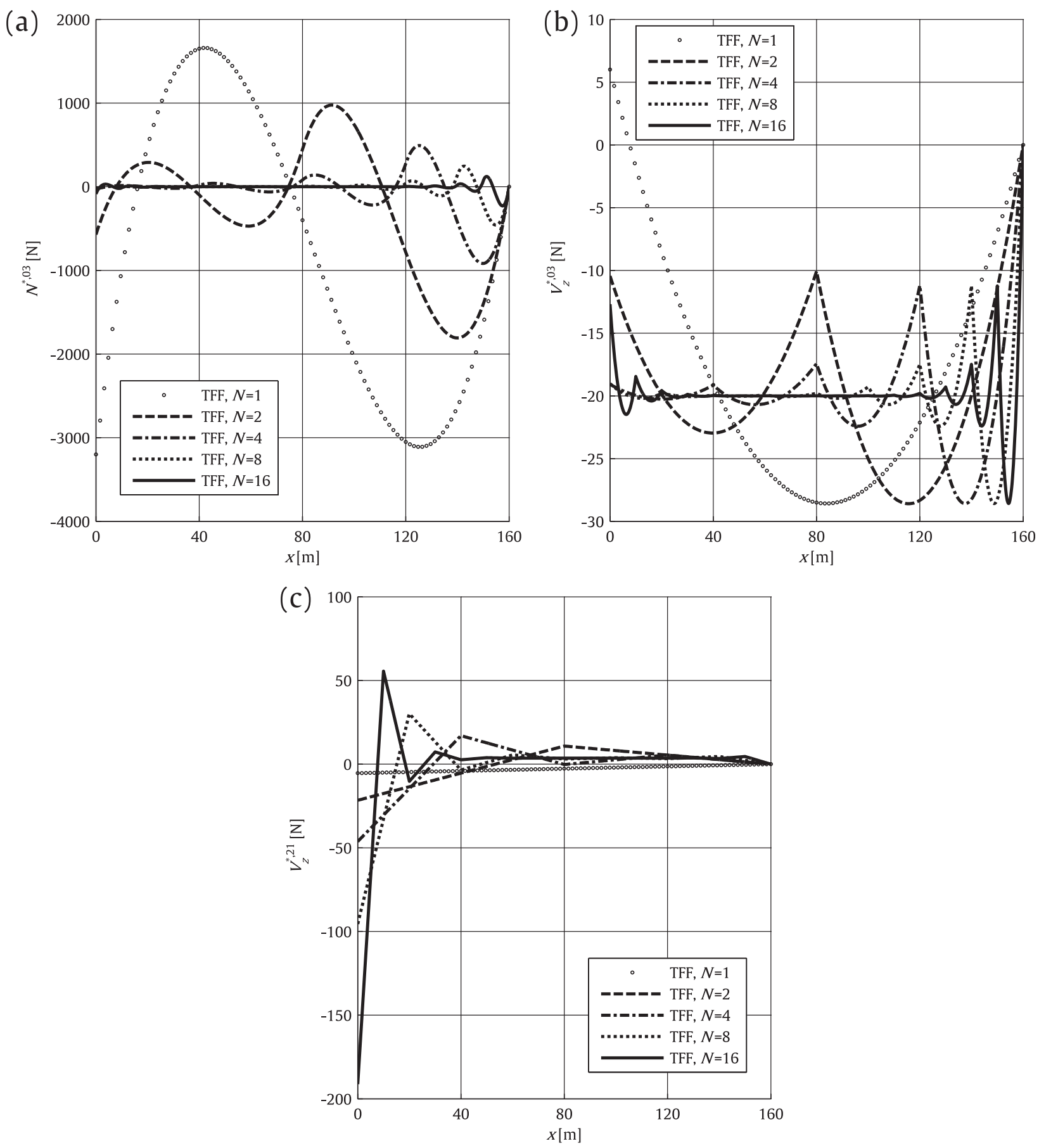

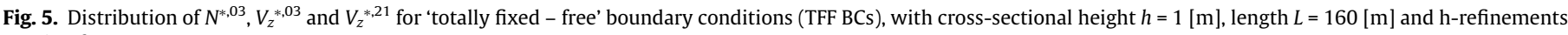
ranging from $N=1$ to $N=16$.

ranging from $N=1$ to $N=16 . V_{z}^{*, 03}$ controls the parabolic distribution of shear stresses $\tau_{x z}$ in the cross-section along the loaded axis, whilst $N^{*, 03}$ commands the corresponding cubic variation of normal stresses $\sigma_{x}$.

However, recalling that the imposed BCs restrict the lateral expansion of the clamped cross-section of the beam, it can be anticipated that-due to Poisson effect-other equilibrium differential equations may be relevant in the element response as well. Namely, the generalised stress-resultants involved in system (7) of Appen$\operatorname{dix} \mathrm{A}$ of the companion paper [1] turn out to be non-negligible; for its special importance, the diagrams of $V_{z}^{*, 21}$-which echo the parabolic distribution of shear stresses along an axis perpendicular to the bending plane-are also shown in Fig. 5. All the generalised stress-resultants of the remaining systems of differential equations are essentially zero.

Figs. 2 and 3 showed that, as the mesh refines, the element level responses-nodal forces and displacements-converge to an asymptotic value. Does that also happen at the cross-sectional level, i.e., regarding the distribution of strains and stresses? The answerand the rationale of the model response-can be looked for in the diagrams of Fig. 5. Their interpretation can be made easier by recalling that the solution of the governing equilibrium differential 
equations is conditioned by the chosen order of specific force approximating polynomials, see Appendix B of Correia et al. [1].

The results given by progressively finer meshes in Fig. 5 ask for the following comments: (i) the refinement is accompanied by a length reduction of the boundary zones where certain higher-order effects associated to the boundary conditions concentrate; (ii) these higher-order boundary effects do not necessarily fade out with refinement-see Fig. 5(c)-and consequently there is no asymptotic value of convergence; (iii) however, outside the aforementioned boundary zones, such convergence does exist and the distributions of the generalised stress-resultants get smoother; for example, Fig. 5(a) and (b) provide clear evidence that $N^{*, 03}$ has a clear trend of convergence towards zero, while $V_{z}^{*, 03}$ stabilises progressively at about $-20[\mathrm{~N}]$, i.e., $-V_{z} / 5$ for $N^{*, 03}=0[\mathrm{~N}]-$ see equation (3) of Correia et al. [1], in Appendix A; a closer look would also indicate that $V_{z}^{*}, 21$ tends to $3.6[\mathrm{~N}]$; (iv) as expected, the diagrams satisfy the zero-valued nodal forces imposed at the free end.

In a simplified way, it can be said that the self-equilibrated solutions provided by the proposed model-inevitably constrained by the imposed BCs and the order of the approximating polynomials-attempt to average the exact equilibrium found away from the boundaries; the latter can be closely reproduced with a refined element mesh.

Fig. 6(a) through (d) show the distribution of normal and shear stresses at the initial clamped cross-section $(x=0[\mathrm{~m}])$ and at midspan $(x=80[\mathrm{~m}])$ for a 1-element mesh. The degree to which the normal and shear stresses deviate respectively from the linear and constant patterns (given by a Timoshenko beam theory) is directly related to the absolute values of $N^{*, 03}$ and $V_{z}^{*, 03}$, as discussed above. In particular, note that the normal stress distribution in Fig. 6(a) is ostensibly nonlinear due to an unrealistically large value of $N^{*, 03}$ at the fixed end; this deviation from linearity reduces drastically at midspan, wherein $N^{*, 03}$ is just a fraction of the previous value. Analogously, $V_{z}^{*, 03}$ and $V_{z}^{*, 21}$ can be held accountable for the shear stress distribution of Fig. 6(c) and (d). The change in the concavity direction about the bending axis $(y)$, associated to the opposite signs of $V_{z}^{*, 03}$ at $x=0[\mathrm{~m}]$ and $x=80[\mathrm{~m}]$, is noteworthy. Eventually, it is observed that the stress distributions of Fig. 6(c), and (d) at a minor degree, also exhibit a curvature perpendicular to the bending axis. Such phenomenon is attributable to the nonzero values of $V_{z}^{*, 21}$.

The distribution of normal and shear stresses at $x=0[\mathrm{~m}]$ and $x=80[\mathrm{~m}]$, for a 16-element mesh, can be found in Fig. 6(e) through (h) for a 16-element mesh. It is immediately evident from Fig. 6(e) and (f) that the distribution of normal stresses does not deviate from linearity, which is a direct consequence of the approximately zero-valued diagram of $N^{*, 03}$. Additionally, the absolute values of the normal stress at the top and bottom fibres of the cross-sections match very closely the results obtained with a classical Euler-Bernoulli beam.

Regarding the shear response, EBBT assumes a null shear strain profile (hence rigid shear behaviour), while the Timoshenko beam theory is based on a constant shear strain pattern. None of the corresponding shear stress distributions are in equilibrium with the evolution of normal stresses. The proposed beam element, on the other hand, produces the shear stress distributions of Fig. 6(g) and $(\mathrm{h})$. The former-instinctively odd in view of the loading direction-can be ascribed to the unrealistically high value of $V_{z}^{*, 21}$ occurring at the clamped base. As discussed above, this is due to a local higher-order effect triggered by the particular BCs defined.

Nevertheless, it should be pointed out that the significant, and somewhat unexpected, contribution of $V_{z}^{*, 21}$ to the shear stress distributions is physically justifiable. In fact, considering both a horizontal and a vertical layer of the beam, due to the changing magnitude of the cross-sectional normal stresses $\sigma_{x}$ along both the beam length and its height, there is a longitudinal variation of the transversal extensions due to Poisson's effect. This leads to distortions related to both shear stresses $\tau_{x z}$ and $\tau_{x y}$, as well as

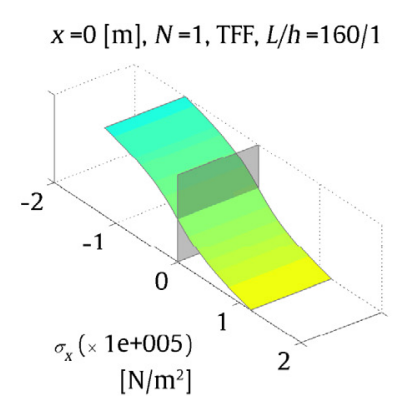

(a)

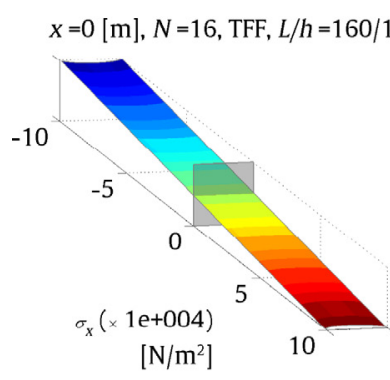

(e)

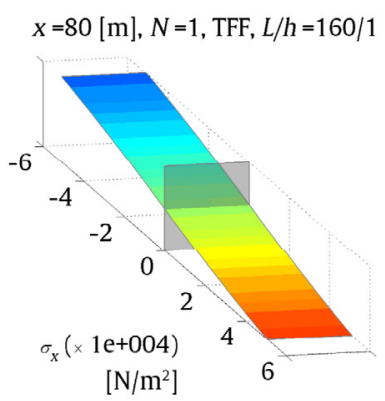

(b)

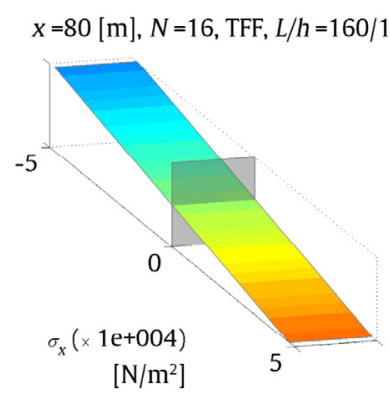

(f)

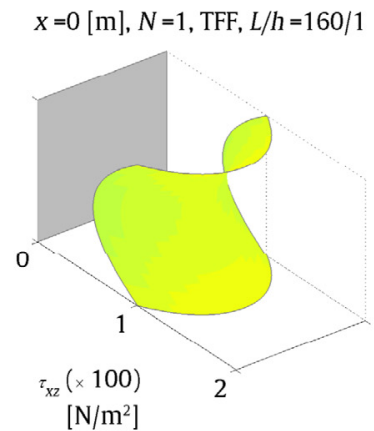

(c)

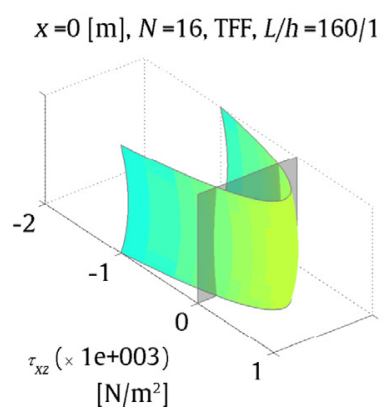

(g)

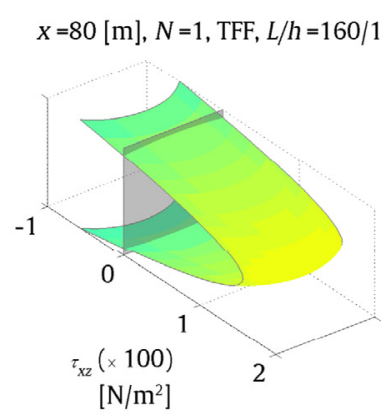

(d)

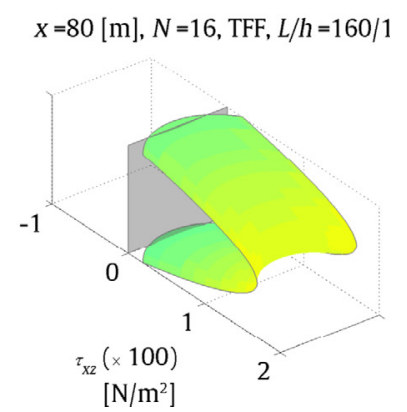

(h)

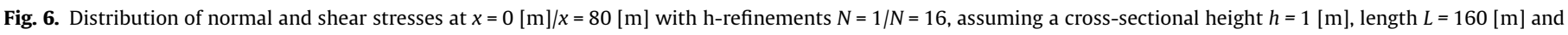
BCS TFF. 
(a)

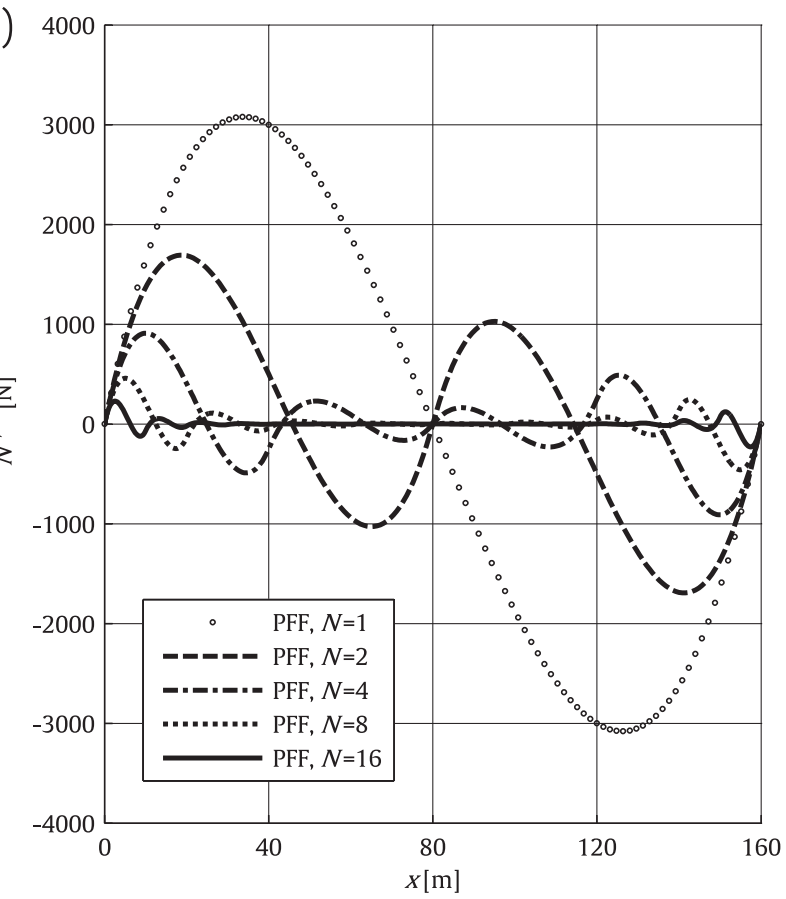

(b)

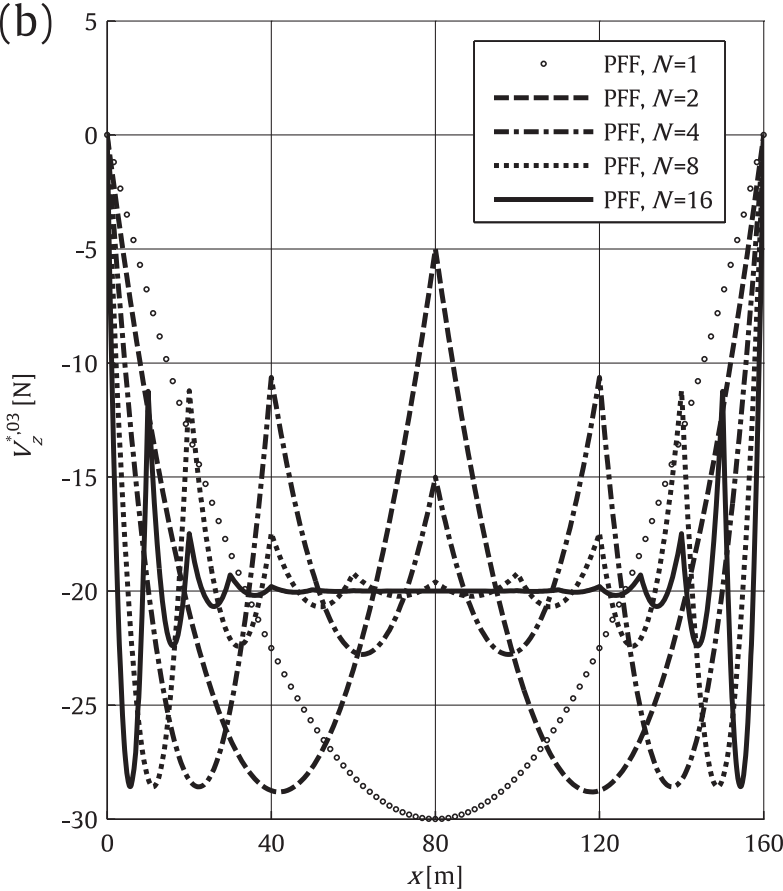

(c)

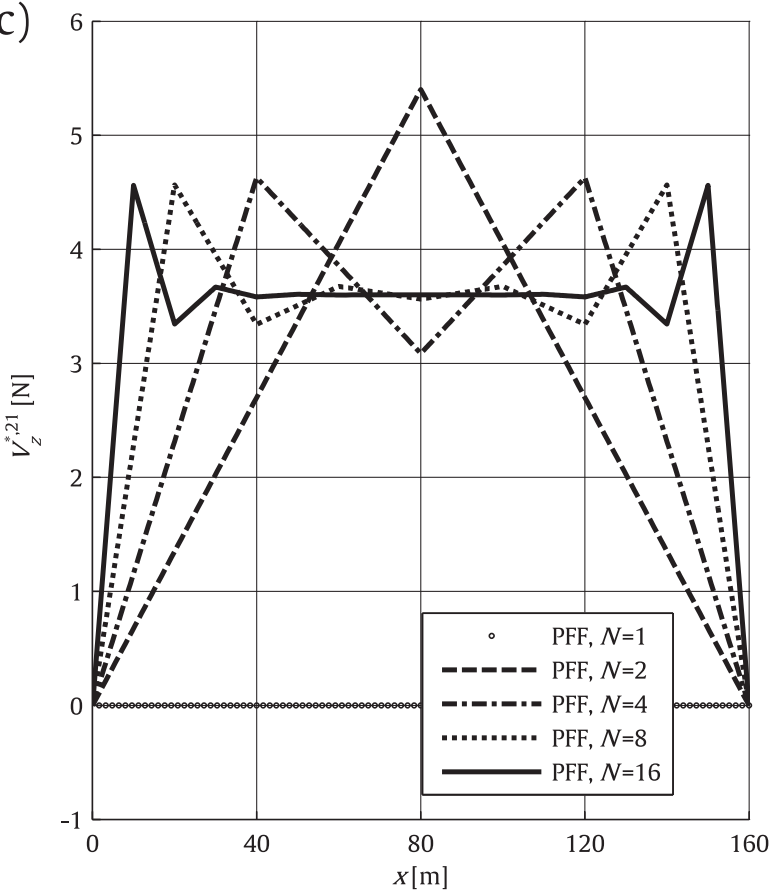

Fig. 7. Distribution of $N^{*, 03}, V_{z}^{*, 03}$ and $V_{z}^{*, 21}$ for 'partially fixed - free' boundary conditions (PFF BCs), with cross-sectional height $h=1$ [m], length $L=160$ [m] and hrefinements ranging from $N=1$ to $N=16$.

to variations of the former along the axis perpendicular to the bending plane, thus originating non-zero values of the generalised stress-resultant $V_{z}^{*, 21}$. Such effect is exacerbated near the clamped end, but exists independently of the BCs, as shown later.

Coulomb, in his paper of 1773 , observed that the effect of shearing forces on the strength of the beam can be neglected if its depth is small in comparison with its length. Later, the rigorous solution of the problem of shearing stresses in beams with simple crosssection geometries was given by Saint-Venant in his famous paper [23]. For more complex cases, the solution put forward by Jouraw- ski (Timoshenko [24]) can be used; it overcomes the limitations of the Euler-Bernoulli and Timoshenko beam theories and allows for the computation of the shear stresses in the cross-section. For a rectangular cross-section as the one in study, their distribution is quadratic, with null values at the top and bottom fibres and a maximum of $\tau_{x z}^{\max }=(3 / 2) V_{z} / A=150\left[\mathrm{~N} / \mathrm{m}^{2}\right]$ attained at mid-height. The results of Fig. $6(\mathrm{~h})$ reliably reproduce it, with a very small allowance for a minor curvature along an axis perpendicular to the bending one, caused by the aforementioned effect represented by $V_{z}^{*, 21}$. 


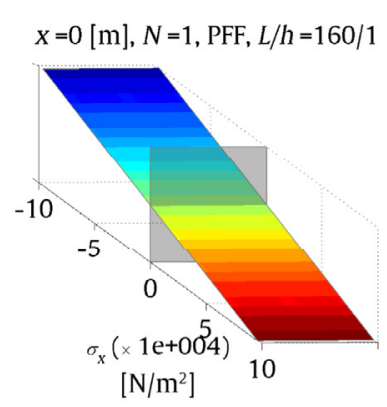

(a)

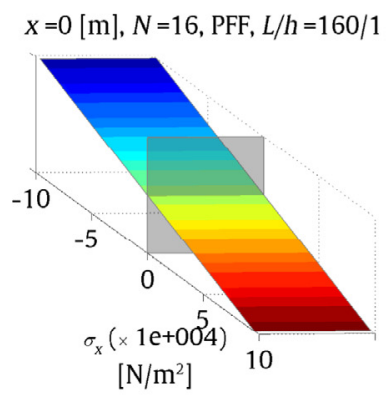

(e)

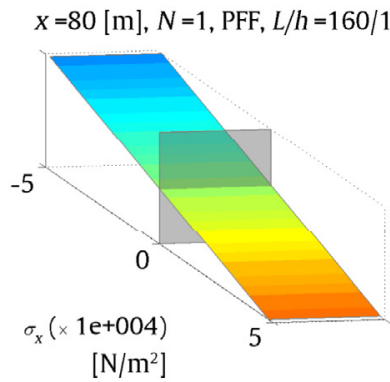

(b)

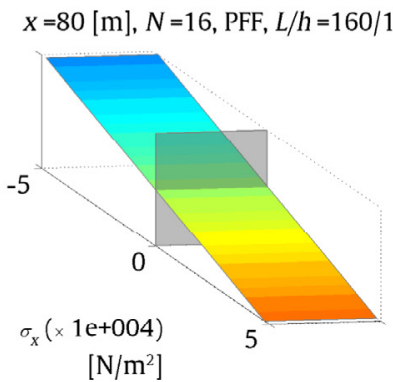

(f)

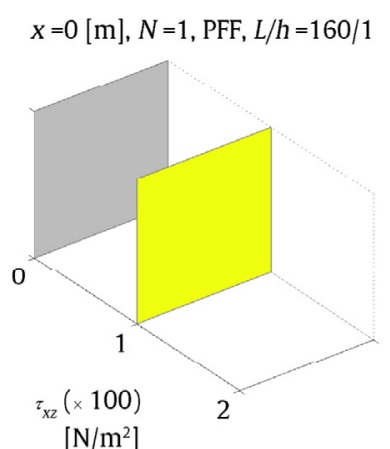

(c)

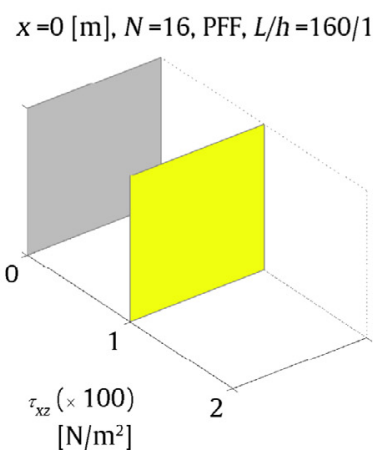

(g)
$X=80[\mathrm{~m}], N=1$, PFF, $L / h=160 / 1$

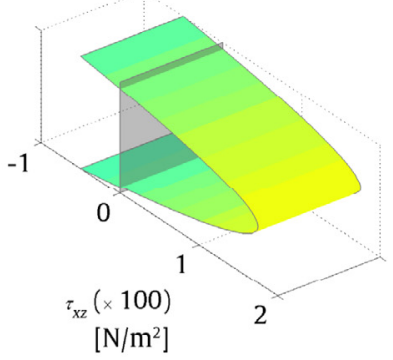

(d)

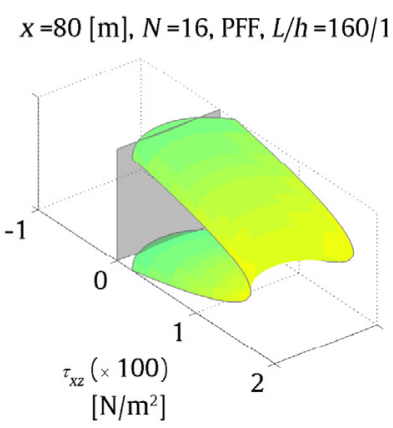

(h)

Fig. 8. Distribution of normal and shear stresses at $x=0[\mathrm{~m}] / x=80[\mathrm{~m}]$ with h-refinements $N=1 / N=16$, assuming a cross-sectional height $h=1[\mathrm{~m}]$, length $L=160[\mathrm{~m}]$ and BCS PFF.

\section{2. 'Partially fixed - free'}

It is noted that the above theoretical solutions do not account for Saint-Venant end effects. Nevertheless, the following questions still arise: is it possible to reproduce exactly Jourawski's stress distributions with the proposed element? If so, which BCs should be adopted? Finally, how does that relates to the minimisation of the local higher-order boundary effects?

As a natural alternative to the previous set of BCs, one could think of the case where, at the clamped extremity, only the six rigid-body displacements corresponding to the Timoshenko beam theory are restrained $\left(u_{x 0}=u_{y 0}=u_{z 0}=\theta_{x}=\theta_{y}=\theta_{z}=0\right)$, leaving the remaining 32 dof as force-controlled and equal to zero. At the free end, the BCs are as in case TFF. For future reference, this new set of $\mathrm{BCs}$ is denoted by the acronym PFF (standing for 'partially fixed free').

Considering such BCs, as expected, both $V_{z}$ and $M_{y}$ show the same evolution of the previous case. The diagrams of $N^{*, 03}, V_{z}^{*, 03}$ and $V_{z}^{*, 21}$ are now included in Fig. 7, while some relevant stress distributions are shown in Fig. 8. The first noticeable difference is the symmetric and antisymmetric behaviour of the generalised stress-resultants along the length, as depicted in Fig. 7, which is an expected feature bearing in mind the imposed BCs. Most importantly, it is noted that as the mesh refines, the results converge to the same asymptotic values of the previous case-naturally, away from the extremity cross-sections wherein the effects of the distinct BCs are noticeable. That is immediately apparent not only for $N^{*, 03}$ and $V_{z}^{*, 03}$, but also for $V_{z}^{*, 21}$-compare Figs. 5(c) and 7(c). Such feature explains the similarity between the plots of Fig. $6(\mathrm{f})$ and $(\mathrm{h})$ and of Fig. $8(\mathrm{f})$ and $(\mathrm{h})$. Another major distinction is that the higher-order effects at the fixed end are now limited by the

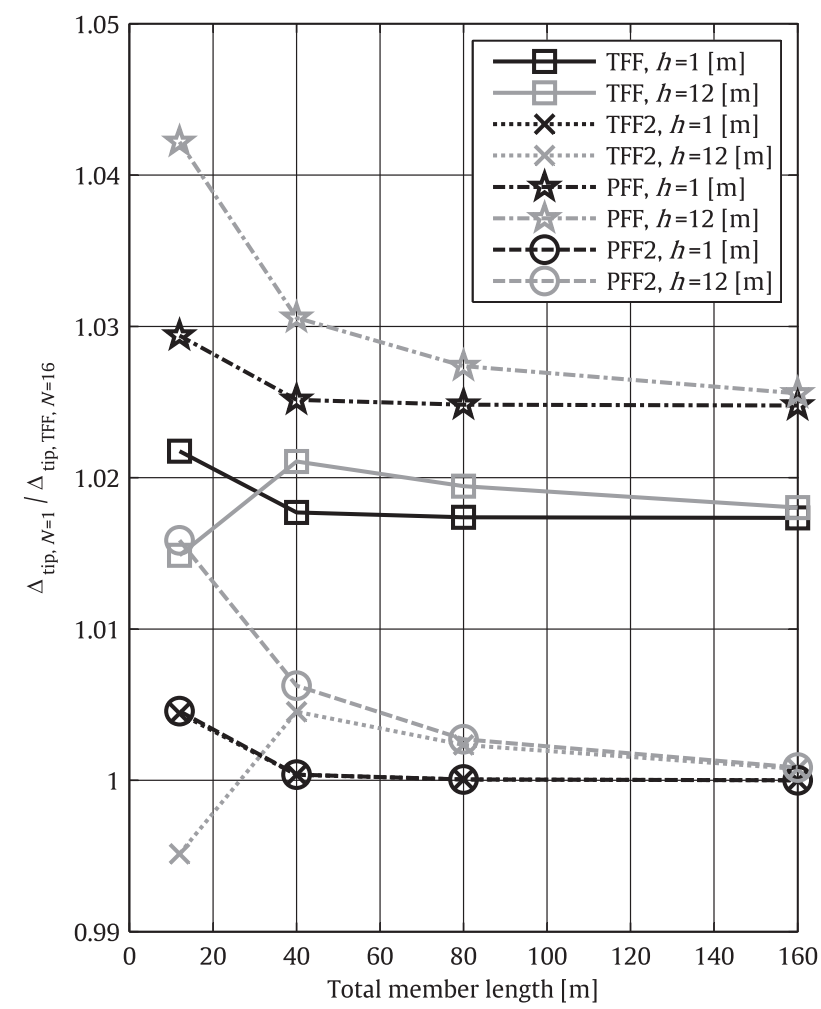

Fig. 9. Influence of the boundary conditions in the accuracy of the tip displacement response given by 1-element meshes with different total lengths and section heights $h$. 

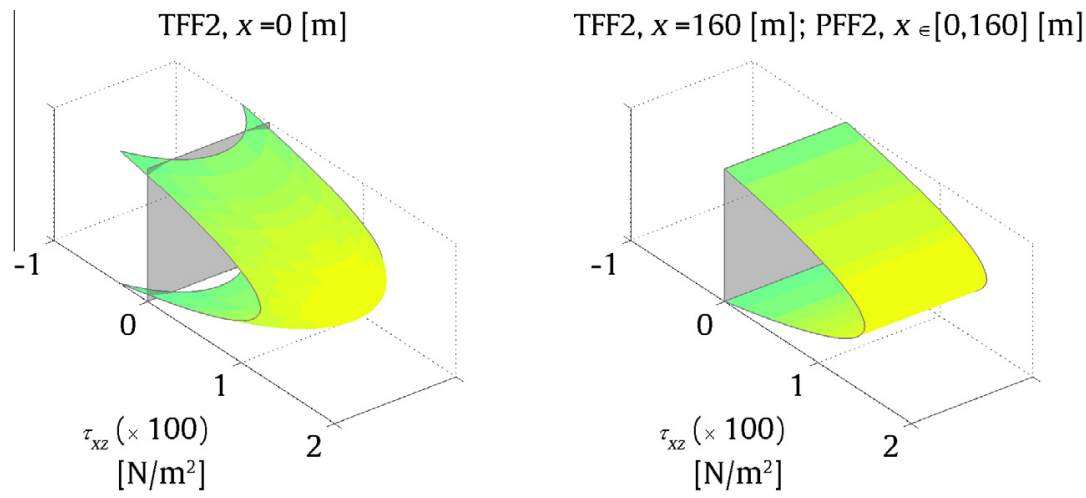

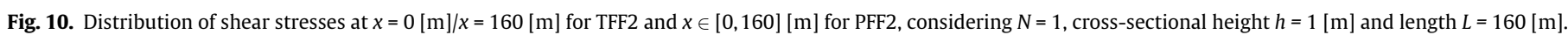

imposed BCs, which explains the constant shear stress distribution at $x=0[\mathrm{~m}]$, as well as the totally linear normal stress profiles at the same location. Figs. 6 and 8 show that the theoretical Jourawski distribution of shear stresses is not exactly reproduced in any cross-section. On the other hand, the two previous cases indicate that $N^{*, 03}$ converges to zero, $V_{z}^{*, 03}$ to about -20 [N] (i.e., $-V_{z} / 5$ for $\left.N^{*, 03}=0[\mathrm{~N}]\right)$, and $V_{z}^{*, 21}$ to $3.6[\mathrm{~N}]$.

\subsection{Other boundary conditions and summary}

It is recalled that the generalised stress-resultant $V_{z}^{*, 21}$ controls the parabolic distribution of shear stresses along the axis perpendicular to the bending plane. Consequently, it must be zero in the Jourawski distribution. Based on the previous rationale, two new sets of BCs are considered: (i) TFF2: it differs from the TFF case in that on the free end a value of $V_{z}^{*, 03}=-20[\mathrm{~N}]$ is imposed alongside with $V_{z}=100[\mathrm{~N}]$; (ii) PFF2: it differs from PFF in that $V_{z}^{*, 03}=$ $-20[\mathrm{~N}]$ is imposed on both ends alongside with $V_{z}=100[\mathrm{~N}]$. Due to space restrictions, the results obtained with these two new sets of BCs are not herein discussed in the same degree of detail as the previous ones but instead in a summarised way.

Fig. 9 shows the influence of all the boundary conditions previously mentioned in the computation of the tip displacement with a 1-element mesh. The ratios plotted therein take the value provided by the 16-element mesh with the original BCs (TFF) as the denominator. Note that Fig. 9 also includes the results for $h=12$ [m], which will be discussed in further detail below. Although the variations between the tip displacements provided by the different BCs may seem relatively minor, they conceal remarkably different element behaviours at the local level. It also stands out that the results provided by the two new sets of BCs, TFF2 and PFF2, clearly provide better results at the global element level than their previous counterparts TFF and PFF.

Fig. 9 also shows that for $h=1$ [m], TFF2 and PFF2 provide an almost identical match at the element level. However, at the cross-sectional level the response is slightly distinct: the distributions of $V_{z}$ and $M_{y}$ are the same as before, as expected; PFF2 recovers exactly-and TFF2 almost exactly-a constant distribution of $V_{z}^{*, 03}=-20[\mathrm{~N}]$ and $N^{*, 03}=0[\mathrm{~N}]$; however, while $V_{z}^{*, 21}=0[\mathrm{~N}]$ for PFF2, TFF2 outputs a linear distribution for $V_{z}^{*, 21}(-5.4[\mathrm{~N}]$ at the fixed end, $0[\mathrm{~N}]$ at the loaded tip).

Fig. 10 shows the shear stress distribution in several cross-sections, confirming that the Jourawski solution is accurately retrieved when the PFF2 BCs are employed. Such conclusion, however, is only valid for the 1-element mesh. It is noted that, based on the self-equilibrated stress-resultants interpolation functions defined in the companion paper, $V_{z}^{*, 21}$ is assumed to be linear along a given element length, thus being inevitably zero when the BCs PFF or PFF2 are considered. In fact, as also shown in Fig. 7(c) for the BCs PFF, although $V_{z}^{*, 21}$ is null for the 1-element mesh, it is different than zero throughout the span when a refined mesh is used, which is a consequence of the real physical behaviour described previously. It results that, for a 16-element mesh, the shear stress distribution at midspan is similar in all cases, corresponding to the one presented in Figs. 6(h) or 8(h).

An exhaustive interpretation of the results for a cross-sectional height $h=12$ [m] is not possible due to length limitations. However, and mainly for comparative purposes with Figs. 5 and 6, the equivalent plots for the case of $L=12$ [m] and $h=12$ [m]-where shear deformations are most pertinent-are plotted in Figs. 11 and 12 . The diagram of $V_{z}^{*, 21}$ is not included for it does not relevantly deviate from zero, since the much reduced normal stresses for this geometry lead to an insignificant influence of the aforementioned Poisson's effects. On the other hand, the distributions of $N^{*, 21}$ and $N^{*, 41}$ are not negligible for $N \geqslant 4$. Figs. 3 and 4 proved the high accuracy of the proposed model at the element level and that the results with a one-element mesh fall very close to the asymptotic value. However, at the cross-sectional level, further inspection is required. One first obvious difference between Figs. 11 and 5 is that, due to the much reduced shear span of the member, the boundary effects stretch their influence along the entire beam length. Fig. 12 depicts the increased shear stress-normal stress interaction, noticeable from the nonlinear pattern of $\sigma_{\mathrm{x}}$ along the bending axis (for $x=0[\mathrm{~m}]$, due to $N^{*, 03}$ ) and along the perpendicular axis (for $N=16$, due to $N^{*, 21}$ and $N^{*, 41}$ ). On the other hand, the distribution of shear stress with $N=1$ are a very good approximation to the ones obtained with $N=16$.

\section{Simply supported beam with distributed loads}

Another major advantage of force-based formulations is that span loads can be directly accounted for, as explicitly derived in the companion paper [1]. The rectangular beam analysed in the two previous sections was also studied by Heyliger and Reddy [2] for simply supported conditions and a uniform load $q=10\left[\mathrm{~N} / \mathrm{m}^{2}\right]$, and is herein used again for comparative purposes. The same values of length $L$ and cross-sectional height $h$ were adopted. Regarding the $\mathrm{BCs}$, on one extremity the three translational displacements, as well as the rotation about the beam longitudinal axis, were restrained; on the other, only the two transversal displacements were blocked. At all the remaining dof the generalised forces were kept equal to zero.

To start with, consider, once again, the cases with cross-sectional height $h=1[\mathrm{~m}]$. Fig. 13 shows the comparison between 

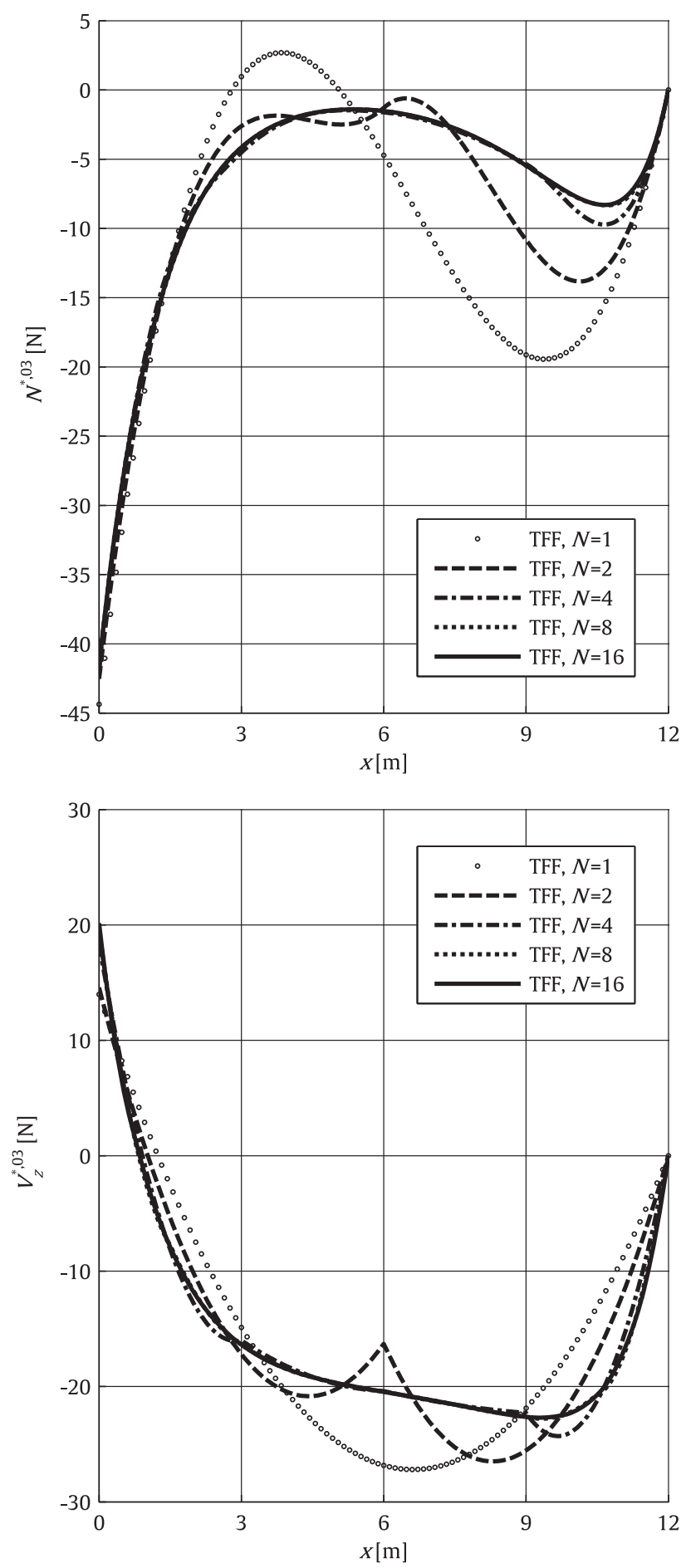

Fig. 11. Distribution of $N^{*, 03}$ and $V_{z}^{*, 03}$ for 'totally fixed - free' boundary conditions (TFF), with cross-sectional height $h=12[\mathrm{~m}]$, length $L=12[\mathrm{~m}]$ and h-refinements ranging from $N=1$ to $N=16$.

the mid-span transversal displacements provided by the present formulation and by that of Heyliger and Reddy [2]. As before, the ordinate axis is normalised with respect to the results given by the EBBT-obtained either analytically or through a force-based model (since a displacement-based one would not provide the exact response). The majority of the comments made in Section 2 regarding Figs. 2 and 3 are again applicable. However, it stands clear that the previously reported accuracy advantage of the proposed model-which was evident for lower number of elements-does not seem so apparent any longer. The same can be said of the not-so-distinct asymptotic values of the two formulations. However, any such comparative comments now fail a basic validity check, since the distributed loading is, in the displacement-based approach, replaced internally by equivalent concentrated loads at both extremities of each element.

In other words, span loads are considered artificially at the nodal level and therefore their effects are not reflected in the distribution of the generalised stress-resultants along the element length. Contrarily, the use of the proposed model allows retrieving exactly the expected distribution of bending moments along the length (parabolic with a maximum of $q L^{2} / 8=32,000[\mathrm{~N} \cdot \mathrm{m}]$ at mid-span) and shear force (linear and symmetric with an absolute value of $q L / 2=800[\mathrm{~N}]$ at the extremities). They are not herein represented since the differences among the different meshes are virtually undetectable with the unaided eye. It is noted that a displacement-based formulation, such as that of Heyliger and Reddy [2], would output incorrect linear and constant distributions of moment and shear force, respectively, within each element. The aforementioned limitations of the displacement-based approach would become more visible if additional types of span loads or different BCs were considered.

It is interesting to observe the influence of the span loads in the evolution of the generalised stress-resultants along the length. The two that most affect the distributions of normal and shear stresses in the several element integration cross-sections, $N^{*, 03}$ and $V_{z}^{*, 03}$, are depicted in Fig. 14. As expected from the physical standpoint, $N^{*, 03}$ tends to zero as the mesh refines, which signals the decreasing deviation of the normal stresses from a cross-sectional linear distribution. Associated with this value, and apart from the known higher-order boundary effects, $V_{z}^{*, 03}$ converges to a linear evolution defined by $-V_{z} / 5$; that is, corresponding to the Jourawski parabolic shear stress distribution.

Finally, Fig. 15 shows the counterpart of Fig. 13 for a cross-sectional height $h=12[\mathrm{~m}]$. The results can be interpreted at the light of what has been discussed immediately above and also in Section 3. It is noted that the shear deformation is now proportionally larger than in the previous cases of concentrated tip load.

\section{Three-dimensional loading and comparison with solid finite elements}

While the previous sections analysed in detail the model performance for a cantilever under in-plane loading, the current one aims at illustrating its three-dimensional behavioural capabilities for a simple imposed unitary torsional rotation at its extremity. For what concerns the cross-sectional geometry, a beam with unitary area is now considered, of height $h=2[\mathrm{~m}]$ and $b=0.5[\mathrm{~m}]$. The mechanical properties of interest are the Young's modulus $E=71.7 \times 10^{9}\left[\mathrm{~N} / \mathrm{m}^{2}\right]$ and the Poisson's ratio $v=0.3$. A squat member of length $L=4[\mathrm{~m}]$ is herein examined, although similar verification tests were also carried out with more slender elements. The BCs involve controlling the 76 generalised nodal displacements, which are all restrained but the one corresponding to the torsional degree of freedom, wherein a unitary value [rad.] is imposed.

Two alternative meshes are used, with one and eight elements. The former outputs a torsional moment of $T=3.472 \times 10^{8}[\mathrm{~N} \cdot \mathrm{m}]$, while the latter produces $T=3.661 \times 10^{8}[\mathrm{~N} \cdot \mathrm{m}]$. The distributions of shear stresses $\tau_{x z}$ and $\tau_{x y}$ at the extremity and midspan crosssections are shown in Fig. 16(a) through (d). It is observed that $\tau_{x z}$ and $\tau_{x y}$ are different than zero at extremities $z= \pm 1$ and $y= \pm 0.25$, respectively, which does not correspond to the exact solution. However, as noted before, the model verifies a higher-order form of equilibrium exactly, i.e., the generalised stress-resultants involved in the complex system of coupled 


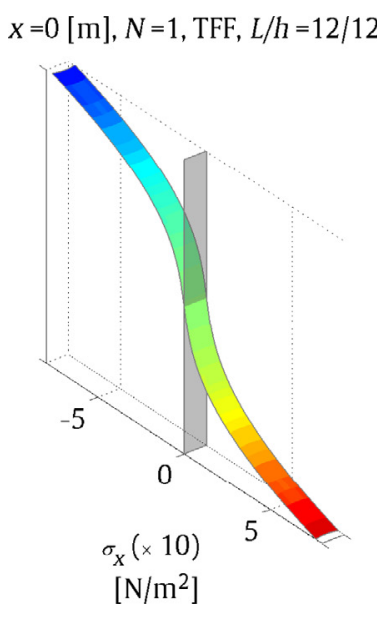

(a)

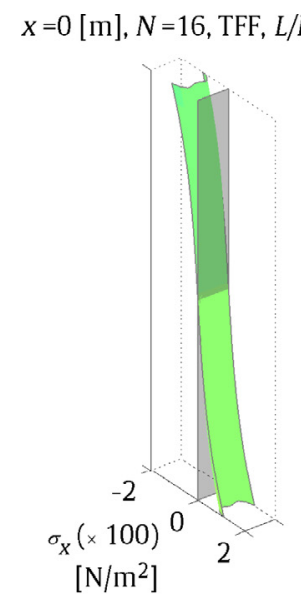

(e)

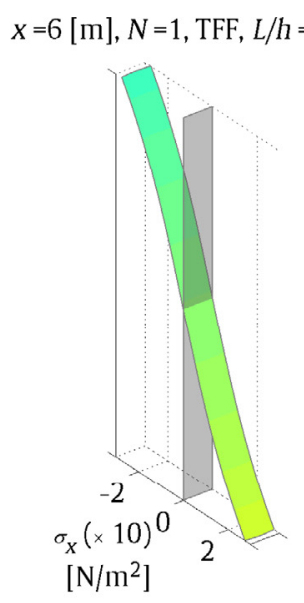

(b)

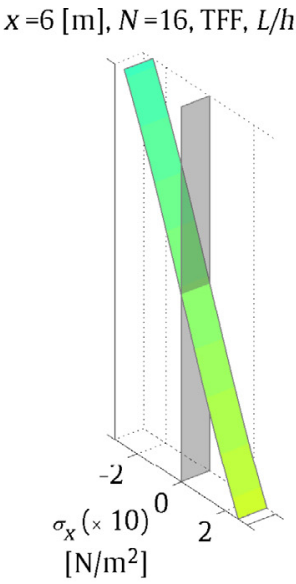

(f)

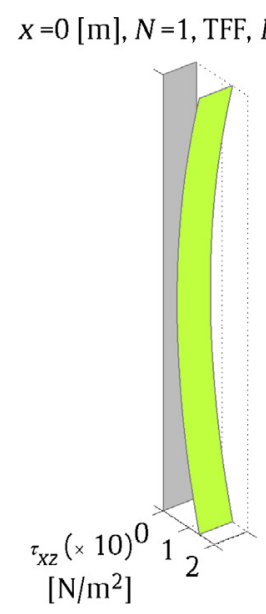

(c)

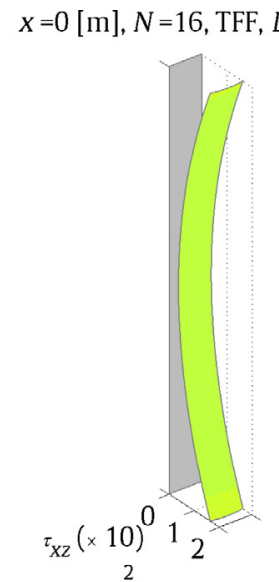

(g)

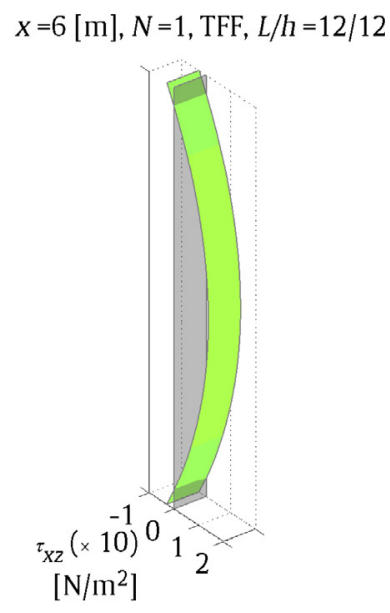

(d)

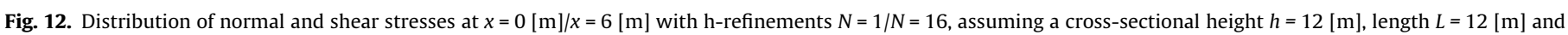
BCS TFF.

differential equations (4) depicted in the Appendix A of Correia et al. [1] are strictly equilibrated. Moreover, if additional cross-sectional deformation modes were to be considered, that condition would be progressively satisfied (and not just in the average sense throughout the section).

Due to the fact that warping is variable since it is restrained at the extremities and different from zero throughout the span, it is expected that normal stresses $\sigma_{x}$ are also non-null and significant-see Fig. 16(e) and (f), corresponding to the case of non-uniform torsion. This figure also shows the influence of different mesh refinements on the absolute value of the obtained normal stresses. Note that, similarly to the flexural-shear interaction examples, these stresses at the extremities are highly influenced by the localised forces which appear due to the boundary conditions.

Consider now the three-dimensional solid finite element model with the coarse and fine meshes depicted in Fig. 16(g) and (h), with 5,535 and 28,413 degrees of freedom respectively, which compare with 76 and 342 for the one and eight-element meshes used in the proposed HOBT. A unitary rotation [rad.] is again imposed and the torsional moment resulting from the lateral joint reactions is computed, yielding values of $T=3.667 \times 10^{8} \quad[\mathrm{~N} \cdot \mathrm{m}]$ and $T=3.613 \times 10^{8}[\mathrm{~N} \cdot \mathrm{m}]$, respectively. The previous results compare very well with the outcome of the present beam model, the differences becoming virtually negligible for more slender elements (not reported herein). It is recalled that the vast majority of proposed beam theories is unable to straightforwardly predict the response of an element under non-uniform torsion.

\section{Comparison with other refined higher-order beam theories}

An assessment of the accuracy of the proposed model can be better ascertained through comparison with other HOBTs. The choice falls on the so-called "Carrera's Unified Formulation", or CUF, briefly discussed in the introductory section of the companion paper [1], due to its systematic approach and completeness (Carrera and Giunta [3]; Carrera et al. [4,25]).

\subsection{Theoretical considerations}

Similarly to the present theory, the displacement field of the CUF is also decomposed into an expansion of components along the axis of the beam and in the cross-section, see Eq. (14) of the companion paper [1]. More specifically, it can be expressed as:

$\mathbf{u}(x, y, z)=\sum_{i=0}^{n} \sum_{j=0}^{i} y^{i-j} z^{j} d_{\frac{(i+1)}{2}+j+1}(x)=\mathbf{U}(y, z) \mathbf{d}(x)$ 


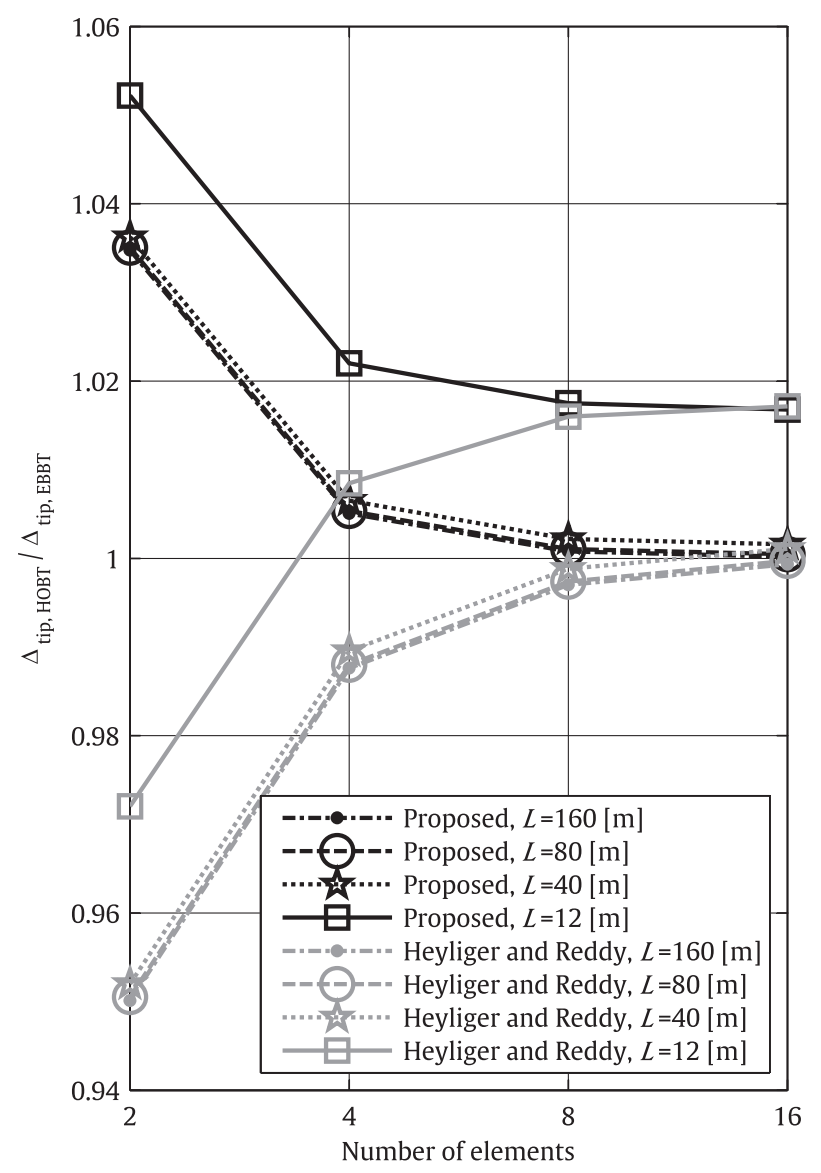

Fig. 13. Comparison of the proposed theory and that of Heyliger and Reddy [2], relative to the results provided by the Euler-Bernoulli theory, for a cross-sectional height of $h=1[\mathrm{~m}]$ and uniform span loads.

where $\mathbf{U}(y, z)$ is the matrix of the displacement interpolation functions and $\mathbf{d}(x)$ are the generalised displacements. It is clear from the previous equation that, just as the interpolation functions of the proposed theory are based on normalised Legendre polynomials, those of the CUF rest on MacLaurin's polynomials.

An illustrative comparison between both models is shown in Table 1 regarding the total number of generalised displacements for $n=1$ to 4 (given by $3(n+1)(n+2) / 2$ for the CUF and by $(n+1)(3 n+1)$ for the proposed HOBT), as well as their corresponding partitioning in components of the displacement field. Within parentheses one can also find the number of terms selected to carry out the derivations in the companion paper and the current numerical examples; in order to proceed with an approximately fair comparison between both theories, the case of $n=4$ was chosen for the CUF.

One interesting feature of the CUF is that the finite element formulation is developed and presented in terms of a systematic 'fundamental nucleo' (Carrera et al. [4]) that it is applicable to any arbitrarily large value of $n$, i.e., it does not require assumptions on the approximation order. Hence, it is possible to immediately obtain any higher-order beam theory.

Nevertheless, since the derivation is based on the principle of virtual displacements, the CUF thus shares the same drawbacks of the theories belonging to the family of displacement-based approaches. In particular, shear-locking shows up-which is therein corrected through selective integration (Bathe [26])-and span loads cannot be included in a straightforward way. Shear locking is avoided with force-based formulations, which
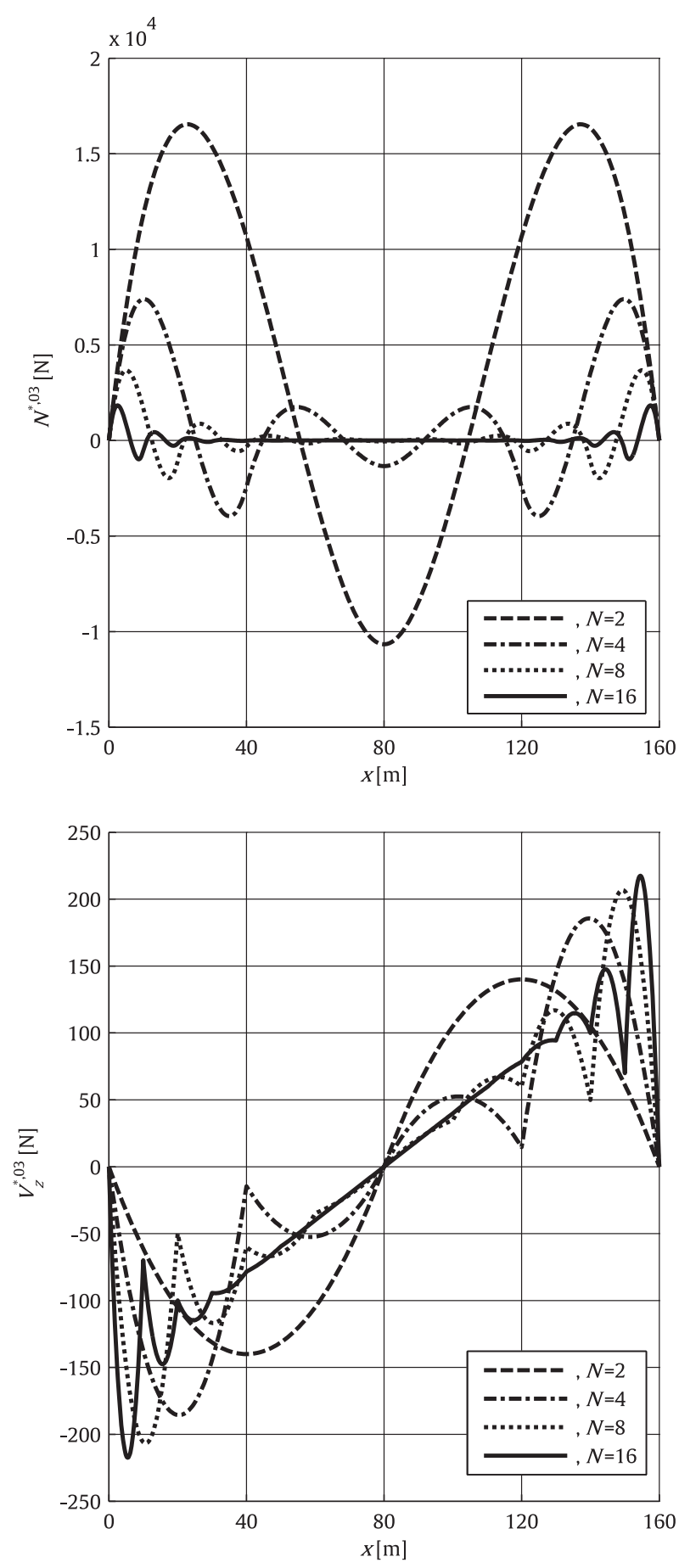

Fig. 14. Distribution of $N^{*, 03}$ and $V_{z}^{*, 03}$ due to uniform loading on a simply supported beam, with cross-sectional height $h=1[\mathrm{~m}]$, length $L=160[\mathrm{~m}]$ and hrefinements ranging from $N=2$ to $N=16$.

additionally allow for an explicit inclusion of distributed loads as discussed in the companion paper [1] and illustrated in the previous section.

However, the essential distinctive feature of the flexibility formulations-herein used for the first time in association with HOBTs-is that beam equilibrium is strictly verified along the element length; this characteristic leads to a fundamentally different structural behaviour that is reflected on a much-improved accuracy of the response at the element level. Section 6.2 provides numerical evidence of such performance. 


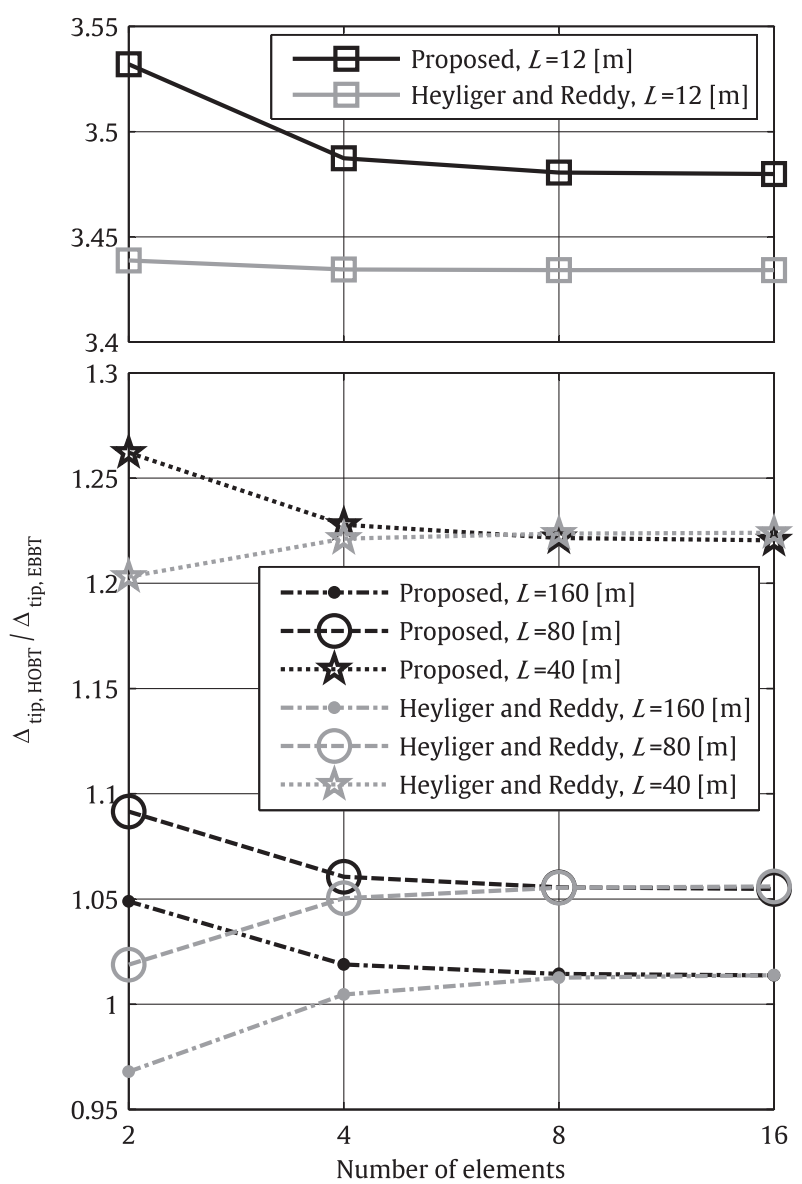

Fig. 15. Comparison of the proposed theory and that of Heyliger and Reddy [2], relative to the results provided by the Euler-Bernoulli theory, for a cross-sectional height of $h=12[\mathrm{~m}]$ and uniform loading.

Another significant aspect differentiates both approaches: the cross-sectional displacement interpolation functions are now set as orthogonal (see Fig. 1), contrary to what occurs in the CUF, thus enabling the definition of non-ambiguous generalised stress-resultants, reducing the coupling of equilibrium equations, and leading to more coherent BCs. Finally, it is noted that the present beam element includes only the two extremity nodes, while Carrera et al. [25] developed elements with two, three and four nodes, respectively addressed as B2, B3 and B4.

\subsection{Numerical applications}

Tabulated data with the results of a cantilevered beam subjected to a lateral force $p=50[\mathrm{~N}]$ at the free end can be found in the work by Carrera et al. [25]. The beam cross-sectional dimensions are $b=h=0.2[\mathrm{~m}]$, the Young's modulus is $E=75 \times 10^{9}\left[\mathrm{~N} / \mathrm{m}^{2}\right]$ and the Poisson's ratio is $v=0.3$. Further, two slenderness ratios are studied, $L / h=10$ and $L / h=100$. Regarding the BCs, the set TFF, previously described in Section 3, is used.

A comparison between both models (the CUF uses $n=4$ ) is depicted in Fig. 17, wherein the tip displacements of meshes with variable number of elements are presented. The comments made in Section 2 regarding Figs. 2 and 3 can now be re-examined. First off, it is noted that the slenderness ratio $L / h=10$ is not small enough-in relation to that of $L / h=100-$ so as to generate a contribution of the shear deformation that is sufficiently significant to change the overall behavioural pattern between Fig. 17(a) and (b). In fact, the displacement provided by the EBBT coincides with the asymptotic values in both cases $\left(1.333 \times 10^{-5}[\mathrm{~m}]\right.$ for $L / h=10$ and $1.333 \times 10^{-2}[\mathrm{~m}]$ for $\left.L / h=100\right)$.

Secondly, and once again, the upper and lower-bounding of the solution-respectively for force-based and displacement-based formulations-stands out. The third remark is that the asymptotic values for both formulations are now seemingly the same, which can be attributed to the fact that a similar contribution of higher order terms takes place, i.e., the corresponding governing equations describe an approximately equivalent structural response. Finally, the perhaps most obvious and simultaneously more important observation: the results given by the proposed HOBT with a very coarse mesh (even with only one single element) are very close to the asymptotic response, while the CUF requires a considerably more refined mesh to attain a comparable degree of accuracy. Note that, for the single element case, the proposed beam has a total of 76 dof while CUF elements B2, B3 and B4 require 90, 135 and 180 dof respectively.

Although the fair comparison should be held with the two-node CUF element B2, it is clear that the dominance extends to the three-node and four-node elements B3 and B4 as well. Bearing in mind that both beam theories share a similar number of higherorder terms, the superiority of the force-based formulation is thus established. This assertion leads back and adds to the aforementioned advantages from the theoretical viewpoint, described in the previous section.

\section{Material nonlinear response}

\subsection{Description of nonlinear solution algorithm and material models}

The flowchart of the solution algorithm for nonlinear analyses implemented in the structural analysis code SAGRES is depicted in Fig. 18. The definition of the variables can be found in the companion paper [1]; further, $x_{m}$ corresponds to the position of each integration section along the element length and $w_{m}\left(x_{m}\right)$ to the associated integration weight, while $\mathbf{s}_{\text {resisting }}^{*}$ represents the resisting normalised stress-resultants. The classical Newton-Raphson procedure was used as the nonlinear solution method at the structural level. The superscript $k$ refers to the load step, while $i$ relates to the Newton-Raphson iteration. Convergence is achieved when the Euclidean norm of the vector with the out-of-balance force components is smaller than a specified threshold (criterion of absolute tolerance) [26]. The developed software also features an automatic sub-stepping technique for those load steps where convergence is not attained.

At the element-section level, the nested state determination of Spacone et al. [27] is used, in which residual deformations $\mathbf{e}_{\text {residual }}^{*}$ (computed from the sectional unbalanced forces $\mathbf{s}_{\text {unbalanced }}^{*}$ ) are transferred from the section to the element level. The corresponding iterations are represented by the superscript $j$. Convergence is attained by the simultaneous verification of two criteria of relative tolerance: (i) ratio between the Euclidean norm of the residual of basic displacements $\mathbf{v}_{\text {residual }}^{*}$ at each nested iteration and the norm of the increment of basic displacements; (ii) ratio between the current work increment and the initial work increment (energy criterion).

The numerical applications in the following section explore the classical EBBT and the current HOBT in nonlinear analyses, which make use respectively of one-dimensional and multiaxial material models. In order to validate the comparison between those beam theories, it should be possible to relate the distinct material models under specific states of stress and strain. On the other hand, to ease the interpretation of the results, the latter should be as simple as possible. Therefore, the following models have been implemented: (i) a one-dimensional plastic model with kinematic hardening, 


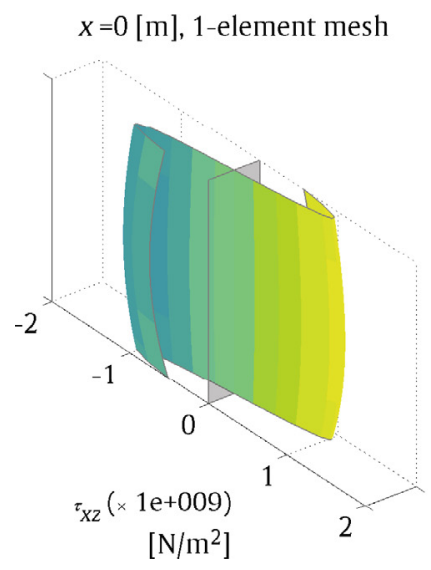

(a)

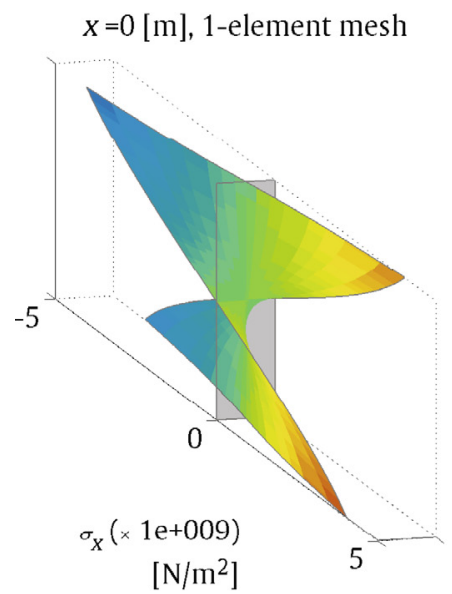

(e)

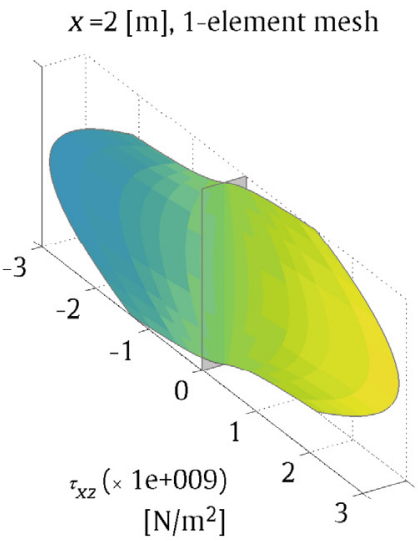

(b)

$X=0[\mathrm{~m}], 8$-elements mesh

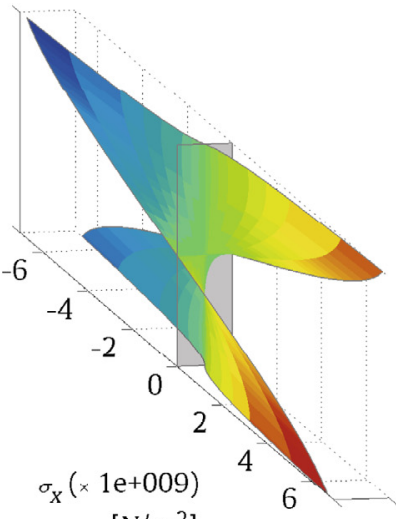

$\left[\mathrm{N} / \mathrm{m}^{2}\right]$
$X=0[\mathrm{~m}], 1$-element mesh

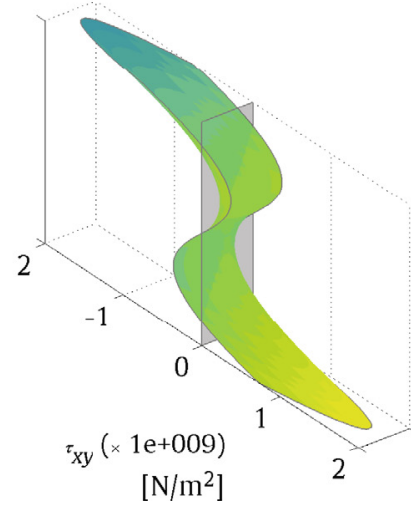

(c)

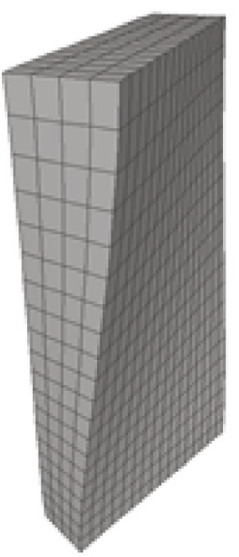

(g)

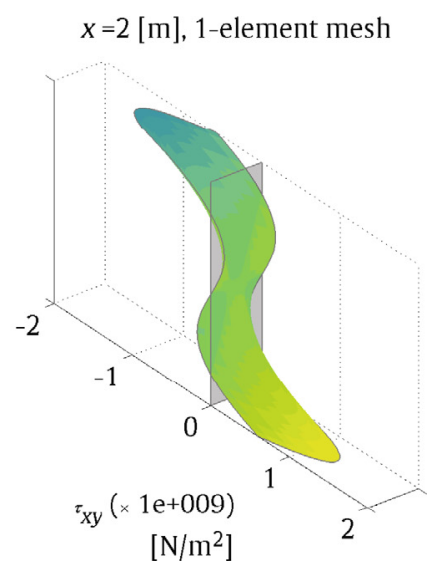

(d)

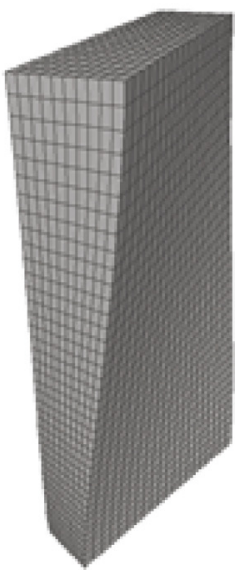

(h)

Fig. 16. (a)-(f): Distribution of shear and normal stresses at different cross-sections; ( $g$ )-(h) Coarse and fine meshes used in the 3D solid FE model.

Table 1

Comparison between the number of terms considered in the CUF formulation and the proposed one.

\begin{tabular}{|c|c|c|c|c|c|c|c|c|}
\hline \multirow[t]{2}{*}{$n$} & \multicolumn{4}{|c|}{ Carrera unified formulation } & \multicolumn{4}{|c|}{ Present formulation, Eq. (13) } \\
\hline & Total & $u_{x}$ & $u_{y}$ & $u_{z}$ & Total & $u_{x}$ & $u_{y}$ & $u_{z}$ \\
\hline 1 & 9 & 3 & 3 & 3 & 8 & 4 & 2 & 2 \\
\hline 2 & 18 & 6 & 6 & 6 & 21 & 9 & 6 & 6 \\
\hline 3 & 30 & 10 & 10 & 10 & 40 & 16 & 12 & 12 \\
\hline 4 & 45 & 15 & 15 & 15 & $65\left(38^{\mathrm{a}}\right)$ & $25\left(16^{\mathrm{a}}\right)$ & $20\left(11^{\mathrm{a}}\right)$ & $20\left(11^{a}\right)$ \\
\hline
\end{tabular}

a Terms adopted in the theoretical derivations and numerical applications.

whose behaviour is defined by the Young's modulus $E$, the uniaxial yield stress $\sigma_{y}^{\text {uniaxial }}$, and the strain hardening ratio $b=E_{p} / E\left(E_{p}\right.$ stands for the plastic modulus); (ii) the multiaxial J2 linear plasticity model described by Auricchio and Taylor [28], which is based on linear evolutionary rules for both the plastic strain and the hardening mechanisms. It is noted that, although this threedimensional model allows for both isotropic and kinematic hardening (defined respectively by the parameters $H_{\text {iso }}$ and $H_{k i n}$ ), the former will not play a role in the subsequent analyses inasmuch as a monotonic loading will be considered; in any case, $H_{\text {iso }}$ will be assigned as zero. The remaining parameters that complete the definition of this model are the initial yield stress in the three-dimensional context $\sigma_{y, 0}$, which relates to $\sigma_{y}^{\text {uniaxial }}$ through $\sigma_{y, 0}=\sqrt{2 / 3} \sigma_{y}^{\text {uniaxial }}$, the shear modulus $G$, and the bulk modulus $K$. The last two parameters will herein be alternatively obtained from the Young's modulus $E$ and the Poisson's ratio $v$.

\subsection{Numerical applications}

The use of the simple material models described above will help to better illustrate the response of the proposed formulation under nonlinear material behaviour. Similarly, the consideration of simple geometrical and loading conditions will facilitate the interpretation of the structural response and the validation of the 

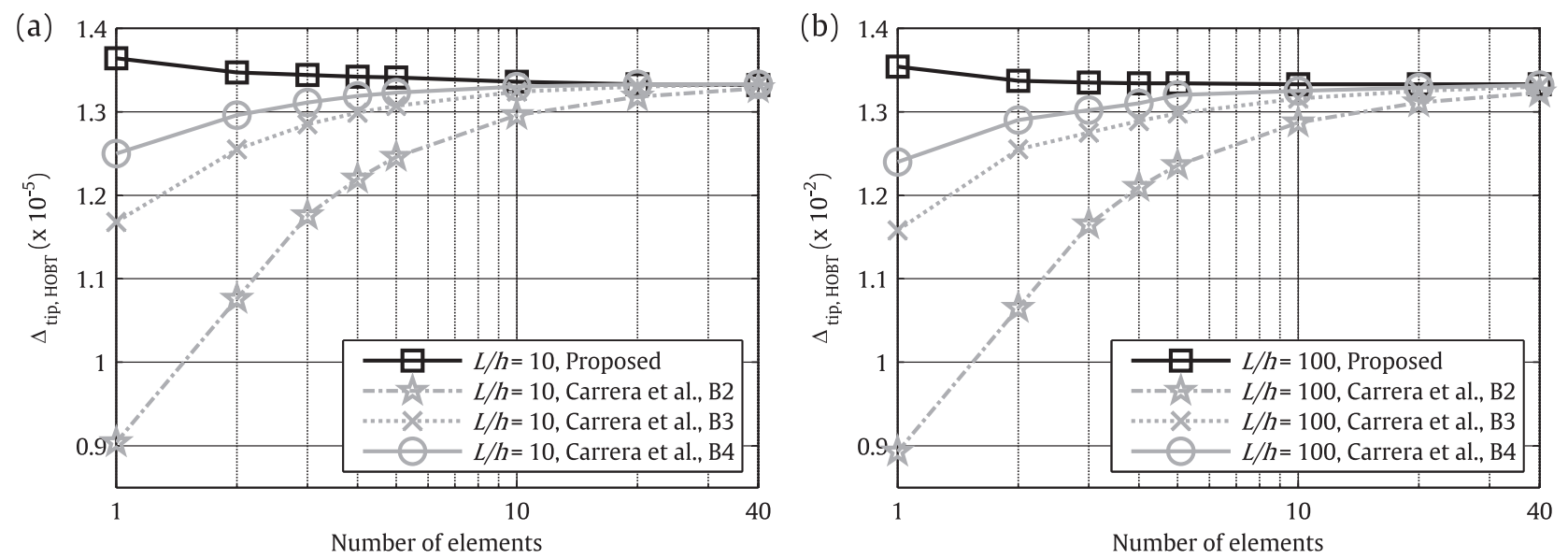

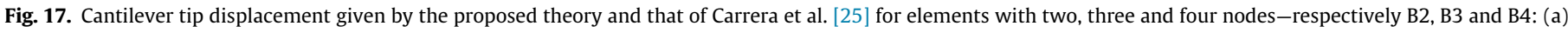
$L / h=10$; (b) $L / h=100$.

developed element. Therefore, the use of the by-now familiar cantilever with in-plane tip load analysed in Sections 2 and 3 appeared as an appropriate choice. In order to put in evidence the particular features of the present approach, a comparison between a forcebased EBBT, whose formulation can be found in Almeida et al. [29], and the current force-based HOBT, is performed. So as to illustrate the simulation of the flexure-shear interaction, the geometrical configuration should be such that expected shear deformation is observable, but on the other hand it should also be relatively small to allow for a meaningful comparison between the two beam theories. In view of such constraints and the results of Fig. 3, a length $L=40[\mathrm{~m}]$ was selected, along with cross-sectional dimensions $h \times b=12 \times 1[\mathrm{~m}]$. The extremity load $p=100[\mathrm{~N}]$ was applied through a suite of 20 load steps-corresponding thus to monotonic increments of $\Delta p=5[\mathrm{~N}]-$ in order to accurately trace the nonlinear response (as explained in Fig. 18). Following the thorough discussion on the effects of boundary conditions and number of elements, the results of a 1-element mesh with the four BCs described in Section 3.3 (designated as TFF, PFF, TFF2, and PFF2) are analysed. As expected, the fixed end for the Euler-Bernoulli beam was simulated by blocking the three translational displacements and the three rotations. The element numerical integration was carried out with a Gauss-Lobatto scheme with eight integration sections (index $m$ of the element sum in Fig. 18). Geometrical nonlinear effects are neglected. The cross-sectional area integration was performed with a grid of $14 \times 4$ (height $\times$ width) quadrilaterals, each one featuring a total of $4 \times 4$ Gauss-Lobatto integration points.

The following material parameters are considered for the unidimensional material used in the force-based EBBT element: $\sigma_{y}^{\text {uniaxial }}=75\left[\mathrm{~N} / \mathrm{m}^{2}\right], E=29,000\left[\mathrm{~N} / \mathrm{m}^{2}\right]$, and $b=0.03$. On the other hand, the currently developed force-based HOBT element employs the parameters for the multiaxial J2 linear plasticity model that follow, which produce the same response under uniaxial states of stress and strain: $\sigma_{y, 0}=\sqrt{2 / 3} \times 75\left[\mathrm{~N} / \mathrm{m}^{2}\right], E=29,000\left[\mathrm{~N} / \mathrm{m}^{2}\right]$, and $H_{\text {kin }}=0.59 \times 10^{3}$; further, the Poisson's ratio is taken as $v=0.3$ and, as stated in the previous section, $H_{\text {iso }}=0$.

Fig. 19 shows the tip force-displacement results obtained with the EBBT and the proposed HOBT with the four different BCs abovementioned. A first immediate observation is that at this global level the results obtained with the HOBT are very similar to those given by the EBBT. The ratio of the corresponding displacements throughout the inelastic response, for the same value of load, is roughly similar to the one obtained previously in Section 2 under elastic response (i.e., approximately 6.5\%, see Fig. 3). However, one could eventually expect to observe a progressive increase in flexibility due to a reduction of shear stiffness with inelasticity. The reasons for such behaviour will be addressed shortly, when the stress distributions in the cross-section are examined. A second comment is that, at this global-level, the distinct BCs do not seem to play a role in the response of the HOBT element. However, as shown below, this apparent agreement masks a different behaviour at the local level. Finally, it should be stated that the response of multiple-element meshes (with two elements, four, eight, etc.) were also analysed and the corresponding force-displacement results are very similar to the 1-element mesh, which explains why they are not depicted in Fig. 19.

The first beneficial feature of the currently proposed HOBT, enabled by the employed force-based formulation, can be observed in the strict verification of equilibrium throughout the member (for example, regarding the distribution of $V_{z}$ and $M_{y}$, which are respectively constant and linear). This holds independently of the degree of inelasticity demand, which would not be reproduced by classical displacement-based formulations.

Fig. 20 shows the distribution of axial strain and axial stress throughout the cross-section at the fixed end. The comparison is carried out between the HOBT (with BCs TFF2) and the EBBT, for $p=100[\mathrm{~N}]$. While the axial strain profiles for both beam theories, as depicted in Fig. 20(a) and (c), are rather similar, the axial stresses, see Fig. 20(b) and (d), depict non-negligible differences. Two phenomena contribute to explain them.

The first one is a physical effect that is compellingly simulated by the proposed force-based HOBT. Namely, the smoothed variation of the axial stress profile at the mid-height of the section (in comparison with the sharp bilinear profile of axial stresses predicted by the EBBT) can be associated to the redistribution of shear stresses along the section with the evolution of plasticity. Fig. 21(a) shows the shear stress distribution while the response is still linear elastic, reproducing closely the Jourawski solution (qualitatively and quantitatively). When the initial effects of plasticity show up, shear is progressively taken by the central part of the section subjected to less plastic demand, in terms of axial stress, as apparent from Fig. 21(b) and (c). At peak force $p=100$ [N], Fig. 21(d) indicates that shear stresses concentrate in a relatively small cross-sectional stripe.

The second one, however, relates to the undesired higher-order effects thoroughly discussed in Section 3, which collaborate to produce the particular nonlinear profile of axial stresses simulated by 


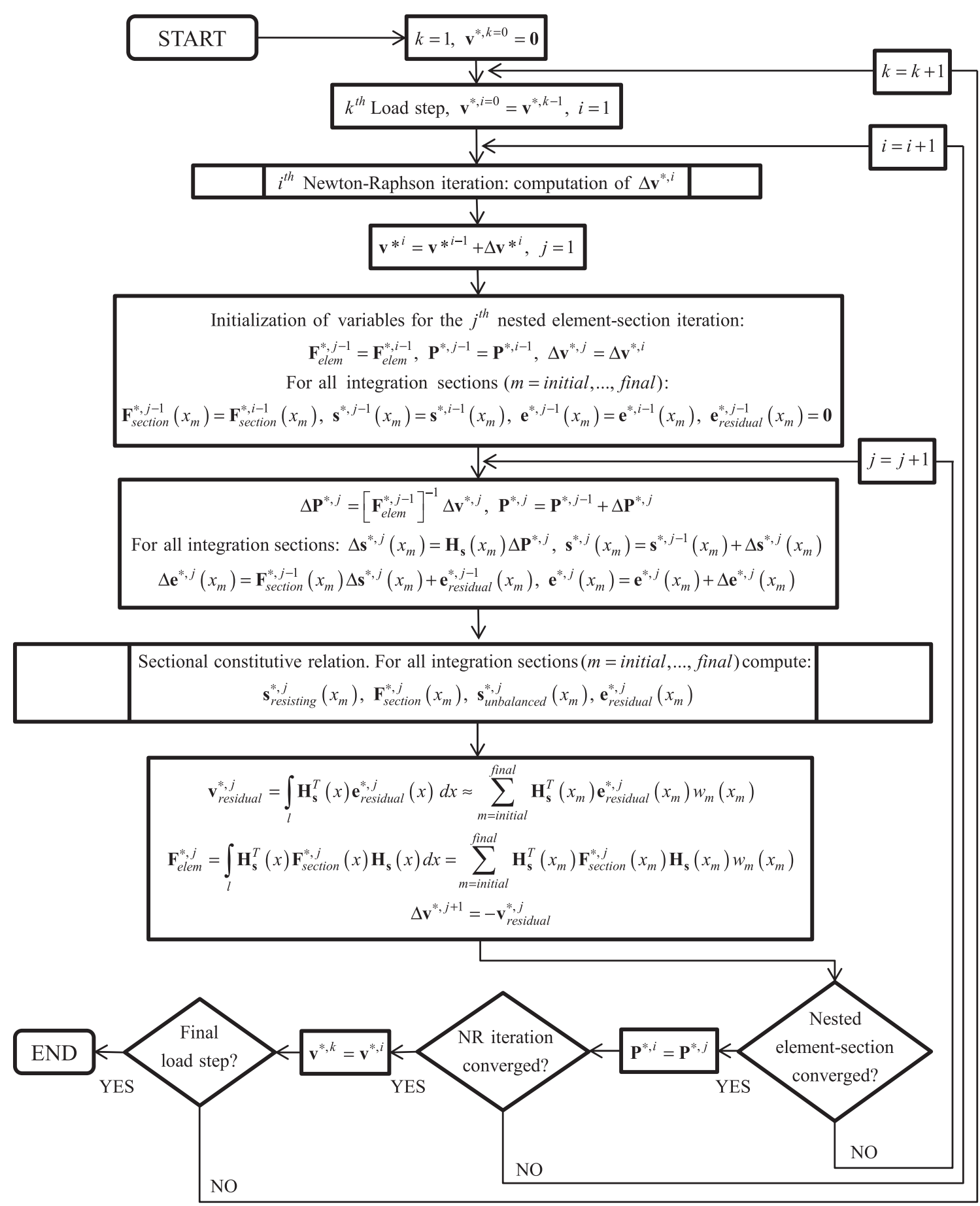

Fig. 18. Flowchart of the implemented solution algorithm for nonlinear analyses.

the HOBT. They are reflected in the appearance of the in-plane stresses shown in Fig. 22. Although roughly one order of magnitude inferior to that of the axial stresses in Fig. 20, they are non-negligible and explain why the maximum axial stress in Fig. 20(b) is approximately $20 \%$ higher than the maximum axial stress predicted by the EBBT in Fig. 20(d), when one could expect that they would instead be smaller. Fig. 22(a) and (b) show that the imposed BCs can affect significantly the importance of these effects. Their minimization deserves further future research.

This example can also be used to provide an indication regarding the computational demand of the proposed formulation. Using the same machine, the higher-order element took approximately 15-20 times (depending on the BCs) more computing time than the classical force-based Euler-Bernoulli element. Bearing in mind that a refined solid finite element mesh with nonlinear material behaviour would take several hundred times more (depending on the software, mesh, material model, solution algorithm, etc.) to run an analogous nonlinear model, it can be said that the present approach occupies a place in the modelling spectrum that is a balance between the pre-existing approaches in terms of output simulation features, as well as considered physical effects, and computing time. 


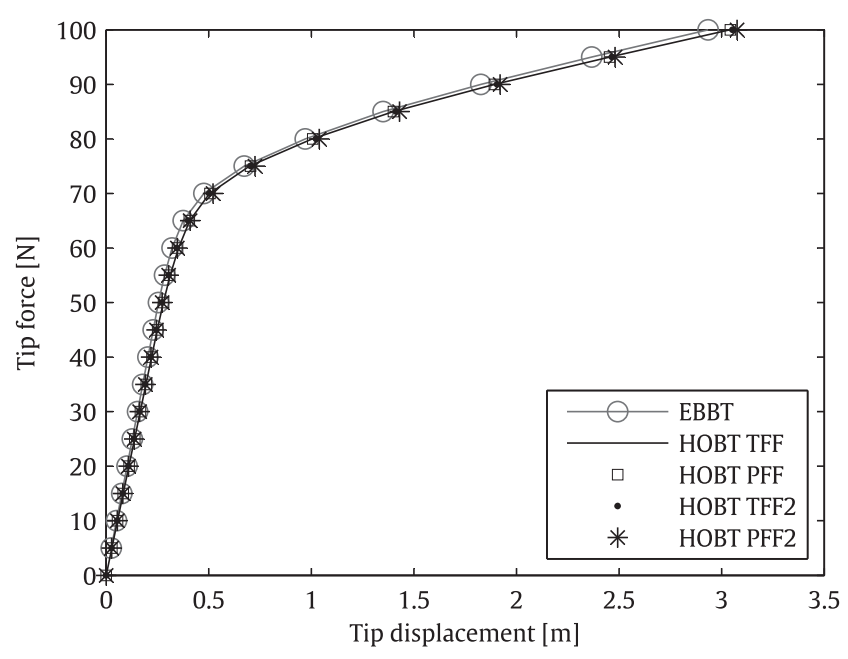

Fig. 19. Inelastic tip force-displacement response of 1-element cantilever modelled with Euler-Bernoulli and the proposed higher-order beam theory with four distinct BCs (TFF, PFF, TFF2, and PFF2).

The simple case-study of this section was meant to show the potential of the present element in the inelastic range. A much more thorough study should be performed to fully explore the possibilities of the theory and the most efficient ways to deal with the unsought higher-order effects associated to the different BCs, which will be addressed in forthcoming publications.

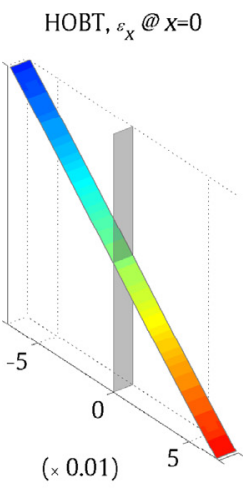

(a)
HOBT, $\sigma_{X} @ X=0$

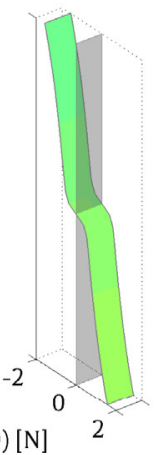

(b)

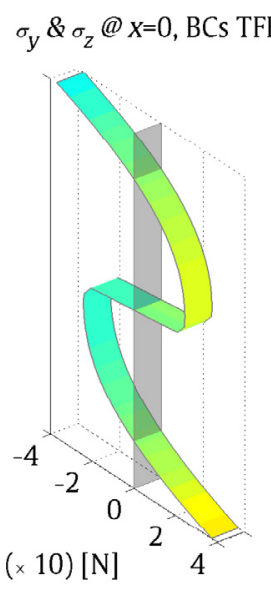

(a)

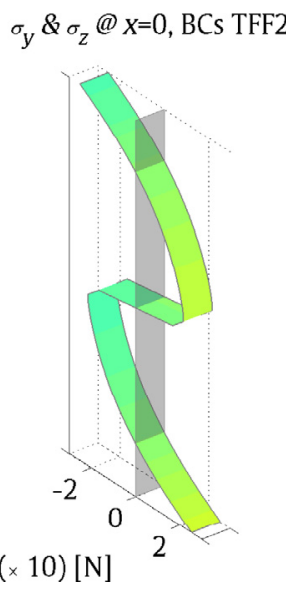

(b)
Fig. 22. Distribution of in-plane stresses at fixed end according to proposed HOBT for $p=100[\mathrm{~N}]$, with BCs: (a) TFF; (b) TFF2.

\section{Conclusions}

The theoretical framework of a beam element that models the flexural-shear-torsional interaction in 3D frames was presented in a companion paper. Its innovative nature resides in the fact that, to the authors' knowledge, a higher-order theory is associated for

Fig. 20. Distribution of axial strains and stresses at fixed end for $p=100[\mathrm{~N}]$ : (a and b) Force-based HOBT with BCs TFF2; (c and d) Force-based EBBT.

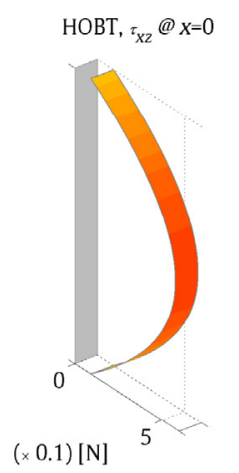

(a)

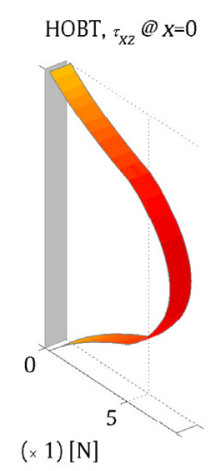

(b)

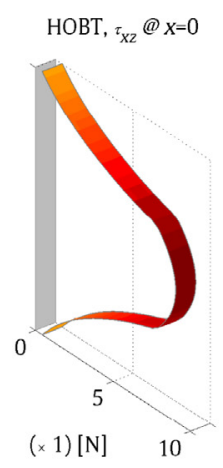

(c)

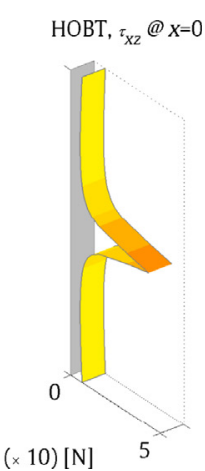

(d)

Fig. 21. Distribution of shear stresses at fixed end for proposed HOBT with BCs TFF2 and: (a) $p=5[\mathrm{~N}]$; (b) $p=60[\mathrm{~N}]$; (c) $p=65[\mathrm{~N}]$; (d) $p=100[\mathrm{~N}]$. 
the first time with a flexibility formulation. The current paper complements the latter, carrying out the corresponding model verification and highlighting its distinguishing features both under linear elastic and nonlinear material response.

Departing from the simple example of a cantilever with inplane tip load, a number of initial comparisons were made. In particular, a distinctive bounding behaviour of the solution was found, which is a feature of formulations based on complementary energy principles. Additionally, the force-based character of the approach also explains the much increased accuracy of the response for coarse meshes. An encouraging match with the output of solid finite element models was obtained.

In classical theories (Euler-Bernoulli or Timoshenko), stressresultants and boundary conditions are of straightforward understanding and definition. However, in a higher-order model, the large number of generalised stress-resultants and displacements-well beyond the six classical ones-are much less intuitive from the engineering standpoint; they relate with the also large number of element nodal displacements and forces that are controlled by appropriate boundary conditions.

The latter reflect the significant adaptability of the model, and consequently physical phenomena that would pass unnoticed with traditional beam theories can thus be retrieved. In particular, the shear deformation, as well as the normal-shear stress interaction, is directly accounted for, which became apparent in the analysis of short span elements. In this context, an appropriate interpretation of the physical meaning of the generalised stress-resultants becomes of paramount importance; this is further emphasised by the uncommon effects that the higher-order boundary conditions can cause on the stress-strain distributions, particularly near the member extremities. These so-called higher-order boundary effects-which also show up in displacement-based formulations, although with distinct traits-play a relevant role in the response. The aforementioned issues were addressed in this paper.

The intrinsic advantages of the proposed model over displacement-based approaches in modelling span loads were also illustrated, and comparisons with other refined higher-order beam theories of similar order demonstrated the outstanding appropriateness of the flexibility formulation. The element was also able to simulate a non-uniform torsional response, reproducing intrinsic three-dimensional features. Comparison with the results from detailed solid models proved encouraging, especially taking into account that the proposed model requires just a fraction of the computing time.

Finally, the performance of the model with a linear plasticity material model is thoroughly analysed and compared with the results from a classical force-based Euler-Bernoulli beam using a one-dimensional plastic material model with kinematic hardening. The element managed to reproduce physical features of member nonlinear behaviour, namely flexure-shear interaction through a progressive redistribution of shear stresses to the part of the cross-section subjected to less plastic demand. Such encouraging results ask for further studies to explore the full potential of the formulation and to simultaneously address the undesired influence of higher-order effects.

\section{Acknowledgements}

The financial support provided by the Portuguese Science and Technology Foundation (FCT) through the 2010 Science and Innovation Operational Programme is gratefully acknowledged. The authors would also like to thank three anonymous reviewers, whose constructive comments helped improving significantly this paper.

\section{References}

[1] Correia AA, Almeida JP, Pinho R. Force-based higher order beam element with flexural-shear-torsional interaction in 3D frames. Part I: Theory. Eng Struct 2015;89:204-17.

[2] Heyliger PR, Reddy JN. A higher order beam finite element for bending and vibration problems. J Sound Vib 1988;126:309-26.

[3] Carrera E, Giunta G. Refined beam theories based on a unified formulation. Int J Appl Mech 2010;02:117-43. http://dx.doi.org/10.1142/S1758825110000500.

[4] Carrera E, Giunta G, Petrolo M. Beam structures: classical and advanced theories. Chichester (UK): John Wiley \& Sons; 2011.

[5] MathWorks, MATLAB - Version 7.9; 2009.

[6] Wackerfuss J, Gruttmann F. A mixed hybrid finite beam element with an interface to arbitrary three-dimensional material models. Comput Methods Appl Mech Eng 2009;198:2053-66. http://dx.doi.org/10.1016/ j.cma.2009.01.020.

[7] Kumar P, Nukala PKVV, White DW. A mixed finite element for threedimensional nonlinear analysis of steel frames. Comput Methods Appl Mech Eng 2004;193:2507-45. http://dx.doi.org/10.1016/j.cma.2004.01.029.

[8] Wackerfuss J, Gruttmann F. A nonlinear Hu-Washizu variational formulation and related finite-element implementation for spatial beams with arbitrary moderate thick cross-sections. Comput Methods Appl Mech Eng 2011;200:1671-90. http://dx.doi.org/10.1016/i.cma.2011.01.006.

[9] Eisenberger M. An exact high order beam element. Comput Struct 2003:81:147-52. http://dx.doi.org/10.1016/S0045-7949(02)00438-8.

[10] Levinson M. A new rectangular beam theory. J Sound Vib 1981;74:81-7.

[11] Reddy JN. Canonical relationships between bending solutions of classical and shear deformation beam and plate theories. Ann Solid Struct Mech 2010;1:9-27. http://dx.doi.org/10.1007/s12356-009-0002-4.

[12] Reddy JN. Theory and analysis of elastic plates and shells. Philadelphia (USA): Taylor and Francis; 2007.

[13] Reddy JN. A simple higher-order theory for laminated composite plates. J Appl Mech 1984;51:745-52.

[14] Almeida JP, Tarquini D, Beyer K. Modelling approaches for inelastic behaviour of RC walls: multi-level assessment and dependability of results. Arch Comput Methods Eng 2014. http://dx.doi.org/10.1007/s11831-014-9131-y.

[15] Washizu K. Variational methods in elasticity and plasticity. 3rd ed. Oxford (UK): Pergamon Press; 1982.

[16] Teixeira de Freitas JA, Moitinho de Almeida JP, Ribeiro Pereira EMB. Nonconventional formulations for the finite element method. Comput Mech 1999;23:488-501. http://dx.doi.org/10.1007/s004660050428.

[17] de Veubeke BF. Displacement and equilibrium models in the finite element method. In: Zienkiewicz OC, Holister GS, editors. Stress Analysis. John Wiley \& Sons; 1965. p. 145-97. http://dx.doi.org/10.1002/nme.339 (Chapter 9).

[18] Pian THH, Tong P. Rationalization in deriving element stiffness matrix by assumed stress approach. In: Proc. 2nd conf. matrix methods struct. analysis. p. 441-69.

[19] Computers and Structures Inc., SAP2000 - Version 16.0.1; 2013.

[20] OpenSees, Open System for Earthquake Engineering Simulation - Version 2.4.3; 2013.

[21] Bickford WB. A consistent higher order beam theory. Dev. Theor. Appl. Mech. 1982;11:137-50.

[22] Prathap G, Vinayak RU, Naganarayana BP. Beam elements based on a higher order theory - II. Boundary layer sensitivity and stress oscillations. Comput Struct 1996;58:791-6.

[23] de Saint-Venant AJCB. Mémoire sur la flexion des prismes. Journal de Mathématiques Pures et Appliquées 1856;Ser. II(1):89-189.

[24] Timoshenko SP. History of strength of materials. New York, USA: McGraw-Hill; 1953 (republished in 1983 by Dover, New York, USA).

[25] Carrera E, Giunta G, Nali P, Petrolo M. Refined beam elements with arbitrary cross-section geometries. Comput Struct 2010;88:283-93. http://dx.doi.org/ 10.1016/j.compstruc.2009.11.002.

[26] Bathe K-J. Finite element procedures. Upper Saddle River, USA: Prentice Hall; 1996.

[27] Spacone E, Ciampi V, Filippou FC. Mixed formulation of nonlinear beam finite element. Comput Struct 1996;58:71-83. http://dx.doi.org/10.1016/00457949(95)00103-N.

[28] Auricchio F, Taylor RL. Two material models for cyclic plasticity: nonlinear kinematic hardening and generalized plasticity. Int J Plast 1995;11:65-98. http://dx.doi.org/10.1016/0749-6419(94)00039-5.

[29] Almeida JP, Das S, Pinho R. Adaptive force-based frame element for regularized softening response. Comput Struct 2012;102-103:1-13. http://dx.doi.org/ 10.1016/i.compstruc.2012.03.018 